\title{
Current literature in mass spectrometry
}

\section{CurRent Awareness - USE the Links online}

\section{View this bibliography online at}

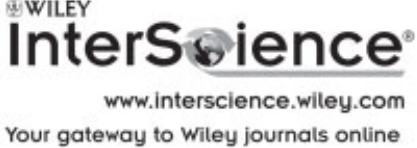

The articles use crossef, the innovative multi-publisher reference linking. This enables readers to move seamlessly from the reference to the cited publication, typically located on a different server and published by a different publisher. Links to MEDLINE abstracts are also available for key journals in the biosciences.

\section{BOOKS, REVIEWS \& SYMPOSIA}

Inductively coupled plasma mass spectrometry (Review)

Beauchemin D// Queens Univ, Dept Chem, Kingston, Ontario, Canada K7L 3N6

Anal Chem 200476 (12) 3395

Protein-nucleic acid interactions and the expanding role of mass spectrometry (Review)

Hanson CL, Robinson CV*// *Univ Cambridge, Dept Chem, Lensfield Rd, Cambridge CB2 1EW, England

J Biol Chem 2004279 (24) 24907

Determination of aminoglycosides and quinolones in food using tandem mass spectrometry: A review

Kotretsou SI// Inst Educat Technol, Food \& Technol Dept, Iokastis 49, GR-10444 Athens, Greece

Crit Rev Food Sci Nutr 200444 (3) 173

Electron-capture mass spectrometry: A powerful tool in biomedical trace level analysis

Leis HJ, Fauler G, Rechberger GN, Windischhofer W// Dept Analyt Biochemistry \& Mass Spectrometry, Auenbruggerpl 30, AT-8036 Graz, Austria

Curr Med Chem 200411 (12) 1585

Structural change in response to ligand binding (Review)

McCammon MG, Robinson CV*// *Univ Cambridge, Chem Lab, Lensfield

Rd, Cambridge CB2 1EW, England

Curr Opin Chem Biol 20048 (1) 60

Mass spectrometry of synthetic polymers (Review)

Peacock PM, McEwen CN*// *DuPont Co Inc, Corp Ctr Analyt Sci, Expt Stn, Wilmington, De 19880, USA

Anal Chem 200476 (12) 3417

Environmental mass spectrometry: Emerging contaminants and current issues (Review)

Richardson SD// US EPA, National Exposure Research Lab, Athens, Ga 30605, USA

Anal Chem 200476 (12) 3337

Surface-enhanced laser desorption ionization time-of-flight mass spectrometry (SELDI TOF-MS) and ProteinChip ${ }^{\circledR}$ technology in proteomics research (Review)

Seibert V, Wiesner A, Buschmann T, Meuer J// EUROPROTEOME AG, Neuendorfstr 24b, DE-16761 Hennigsdorf, Germany

Pathol Res Pract 2004200 (2) 83

Use of AMS in the marine environment (Review)

Skipperud L, Oughton DH// Agr Univ Norway, Dept Soil \& Water Sci, Isotope Lab, POB 5028, NO-1432 As, Norway

Environ Int 200430 (6) 815

\section{INSTRUMENTAL TECHNIQUES \& METHODS}

Influence of droplet size, capillary-cone distance and selected instrumental parameters for the analysis of noncovalent protein-ligand complexes by nanoelectrospray ionization mass spectrometry

Benkestock K, Sundqvist G, Edlund PO, Roeraade J// Biovitrumn AB, Dept Struct Chem, Biovitrum AB, SE-11276 Stockholm, Sweden

J Mass Spectrom 200439 (9) 1059

Desorption/ionization mass spectrometry on nanocrystalline titania sol-gel- deposited films

Chen CT, Chen YC*// *Nat Chiao Tung Univ, Dept Appl Chem, Hsinchu 300, Taiwan

Rapid Commun Mass Spectrom 200418 (17) 1956

Electronic gel protein transfer and identification using matrix-assisted laser desorption/ionization-mass spectrometry

Cooper JW, Gao J, Lee CS*// *Univ Maryland, Dept Chem \& Biochem, College Park, Md 20742, USA

Electrophoresis 200425 (9) 1379

TANDEM: Matching proteins with tandem mass spectra

Craig R, Beavis RC*// *Univ Manitoba, Manitoba Ctr Proteom, Winnipeg,

Manitoba, Canada R3T 2N2

Bioinformatics 200420 (9) 1466

Liquid chromatographic-mass spectrometric separation of oligoalanine peptide stereoisomers: Influence of absolute configuration on enantioselectivity and two-dimensional separation of diastereomers and enantiomers

Czerwenka C, Maier NM, Lindner $\mathrm{W} * / /$ *Univ Vienna, Inst Analyt Chem, Wahringerstr 38, AT-1090 Vienna, Austria

J Chromatogr A 20041038 (1-2) 85

On-line atmospheric pressure matrix-assisted laser desorption/ionization mass spectrometry

Daniel JM, Ehala S, Friess SD, Zenobi R*// *ETH, Dept Chem \& Appl Biosci, CH-8093 Zurich, Switzerland

Analyst 2004129 (7) 574

On-line coupling of high-performance liquid chromatography to a continuousflow enzyme assay based on electrospray ionization mass spectrometry

De Boer AR, Letzel T, Van Elswijk DA, Lingeman H, Niessen WMA, Irth H// Free Univ Amsterdam, Fac Sci, Sect Analyt Chem \& Appl Spect, Dept Chem \& Pharmaceut Sci, NL-1081 HV Amsterdam, The Netherlands Anal Chem 200476 (11) 3155

Determination of glycopeptide structures by multistage mass spectrometry with low-energy collision-induced dissociation: Comparison of electrospray ionization quadrupole ion trap and matrix-assisted laser desorption/ionization quadrupole ion trap reflection time-of-flight approaches

Demelbauer UM, Zehl M, Plematl A, Allmaier G, Rizzi A*// *Univ Vienna, Inst Analyt Chem, Waehringer Str 38, AT-1090 Vienna, Austria Rapid Commun Mass Spectrom 200418 (14) 1575

Aptamer-enhanced laser desorption/ionization for affinity mass spectrometry

In order to keep subscribers up-to-date with the latest developments in their field, John Wiley \& Sons are providing a current awareness service in each issue of the journal. The bibliography contains newly published material in the field of mass spectrometry. Each bibliography is divided into 11 sections: 1 Books, Reviews \& Symposia; 2 Instrumental Techniques \& Methods; 3 Gas Phase Ion Chemistry; 4 Biology/Biochemistry: Amino Acids, Peptides \& Proteins; Carbohydrates; Lipids; Nucleic Acids; 5 Pharmacology/Toxicology; 6 Natural Products; 7 Analysis of Organic Compounds; 8 Analysis of Inorganics/Organometallics; 9 Surface Analysis; 10 Environmental Analysis; 11 Elemental Analysis. Within each section, articles are listed in alphabetical order with respect to author. 
Dick LW, McGown LB*// *Duke Univ, Dept Chem, Box 90346, Durham, NC 27708, USA

Anal Chem 200476 (11) 3037

Controlling matrix suppression for matrix-assisted laser desorption/ionization analysis of small molecules

Donegan M, Tomlinson AJ, Nair H, Juhasz P// Applied Biosystems, 500 Old Connecticut Path, Framingham, Ma 01701, USA

Rapid Commun Mass Spectrom 200418 (17) 1885

Quantitative analysis of tryptic protein mixtures using electrospray ionization Fourier transform ion cyclotron resonance mass spectrometry

Hagman C, Ramstrom M, Hakansson P, Bergquist J*// *Univ Uppsala, Inst Chem, Dept Analyt Chem, Box 599, SE-75124 Uppsala, Sweden

J Proteome Res 20043 (3) 587

MS1, MS2 and SQT - Three unified, compact, and easily parsed file formats for the storage of shotgun proteomic spectra and identifications

Hayes McDonald W, Tabb DL, Sadygov RG, MacCoss MJ, Venable J, Graumann J, Johnson JR, Cociorva D, Yates JR*// *Scripps Res Inst, Dept Cell Biol, 10550 Nth Torrey Pines Rd, La Jolla, Ca, USA

Rapid Commun Mass Spectrom 200418 (18) 2162

Size-selective extraction of peptides from urine for mass spectrometric analy-

Hortin GL, Meilinger B, Drake SK// NIH, Dept Lab Med, Warren G Magnuson Clin Ctr, Bldg 10, Bethesda, Md 20892, USA

Clin Chem 200450 (6) 1092

Matrix-assisted laser desorption/ionization time-of-flight mass spectrometry as a tool for fast identification of protein binders in color layers of paintings Hynek R, Kuckova S, Hradilova J, Kodicek M// Inst Chem Technol, Dept Biochem \& Microbiol, Technicka 5, CZ-16628 Prague 6, Czech Republic Rapid Commun Mass Spectrom 200418 (17) 1896

Efficacy and compatibility with mass spectrometry of methods for elution of proteins from sodium dodecyl sulfate-polyacrylamide gels and polyvinyldifluoride membranes

Jorgensen CS, Jagd M, Sorensen BK, McGuire J, Barkholt V, Hojrup P, Houen G*// *Statens Serum Inst, Dept Res \& Dev, DK-2300 Copenhagen, Denmark

Anal Biochem 2004330 (1) 87

Photoionization pathways and free electrons in UV-MALDI

Knochenmuss R// Novartis Inst Biomed Res, WSJ 503.11.04, CH-4002 Basel, Switzerland

Anal Chem 200476 (11) 3179

Silylation of acrylamide for analysis by solid-phase microextraction/gas chromatography/ion-trap mass spectrometry

Lagalante AF, Felter MA// Villanova Univ, Dept Chem, 800 Lancaster Ave, Villanova, Pa 19085, USA

J Agric Food Chem 200452 (12) 3744

Highly informative proteome analysis by combining improved $\mathrm{N}$-terminal sulfonation for de novo peptide sequencing and online capillary reverse-phase liquid chromatography/tandem mass spectrometry

Lee YH, Kim MS, Choie WS, Min HK, Lee SW*// *Korea Univ, Dept Chem, 1,5-Ka, Anam dong, Seoul 136 701, South Korea

Proteomics 20044 (6) 1684

RScore: A peptide randomicity score for evaluating tandem mass spectra Li F, Sun W, Gao Y, Wang J// Chinese Acad Sci, Inst Automation, Lab Complex Systems \& Intelligence Sci, CN-100080 Beijing, People's Rep China Rapid Commun Mass Spectrom 200418 (14) 1655

Flow-rate characterization of microfabricated polymer microspray emitters Lion N, Gellon JO, Girault HH*// *Ecole Polytech Fed Lausanne, Lab Electrochim Phys \& Analyt, CH-1015 Lausanne, Switzerland Rapid Commun Mass Spectrom 200418 (14) 1614

Comparison of laser-induced dissociation and high-energy collision-induced dissociation using matrix-assisted laser desorption/ionization tandem time-offlight (MALDI-TOF/TOF) for peptide and protein identification

Macht M, Asperger A, Deininger SO// Bruker Daltonik GmbH, Permoser Str 15, DE-04159 Leipzig, Germany

Rapid Commun Mass Spectrom 200418 (18) 2093

Comparison of two glutaraldehyde immobilization techniques for solid-phase tryptic peptide mapping of human hemoglobin by capillary zone electrophoresis and mass spectrometry

Migneault I, Dartiguenave C, Vinh J, Bertrand MJ, Waldron $\mathrm{KC} * / /$ *Univ Montreal, Dept Chem, CP 6128, Succ Centre-Ville, Montreal, Quebec, Canada H3C $3 \mathrm{~J} 7$

Electrophoresis 200425 (9) 1367

5-Amino-2-mercapto-1,3,4-thiadiazole: A new matrix for the efficient matrixassisted laser desorption/ionization of neutral carbohydrates (Letter)

Mirza SP, Raju P, Madhavendra SS, Vairamani $\mathrm{M} * / /$ *Indian Inst Chem Technol, Natl Ctr Mass Spectrom, IN-500007 Hyderabad, India
Rapid Commun Mass Spectrom 200418 (14) 1666

Thin-layer chromatography combined with diode laser desorption/atmospheric pressure chemical ionization mass spectrometry

Peng S, Ahlmann N, Kunze K, Nigge W, Edler M, Hoffman T, Franzke J*// *ISAS - Inst Analyt Sci, Bunsen-Kirchhoff-Str 11, DE-44139 Dortmund, Germany

Rapid Commun Mass Spectrom 200418 (16) 1803

Continuous-flow sample introduction for field desorption/ionization mass spectrometry

Schaub TM, Linden HB, Hendrickson CL, Marshall AG*// *Ion Cyclotron Resonance Prog, Natl High Magnetic Field Lab, 1800 East Paul Dr, Tallahassee, Fl 32310, USA

Rapid Commun Mass Spectrom 200418 (14) 1641

A critical evaluation of high performance liquid chromatography-electrospray ionisation-mass spectrometry and capillary electrophoresis-electrospray-mass spectrometry for the detection and determination of small molecules of significance in clinical and forensic science

Smyth WF, Brooks P// Univ Ulster, Sch Biomed Sci, Coleraine BT52 1SA, Northern Ireland

Electrophoresis 200425 (10-11) 1413

Time-of-flight secondary ion mass spectrometry (TOF-SIMS): Versatility in chemical and imaging surface analysis

Sodhi RNS// Univ Toronto, Dept Chem Eng \& Appl Chem, Surface Interface Ontario, 200 Coll St, Toronto, Ontario, Canada M5S 3E5

Analyst 2004129 (6) 483

The reproducible acquisition of comparative liquid chromatography/tandem mass spectrometry data from complex biological samples

Stewart II, Zhao L, Le Bihan T, Larsen B, Scozzaro S, Figeys D, Mao GD, Ornatsky O, Dharsee M, Orsi C, Ewing R, Goh R// MDS-Proteomics Inc, 251 Attwell Dr, Toronto, Ontario, Canada M9W 7H4

Rapid Commun Mass Spectrom 200418 (15) 1697

Novel linear quadrupole ion trap/FT mass spectrometer: Performance characterization and use in the comparative analysis of histone $\mathrm{H} 3$ posttranslational modifications

Syka JEP, Marto JA, Bai DL, Horning S, Senko MW, Schwartz JC, Ueberheide B, Garcia B, Busby S, Muratore T, Shabanowitz J, Hunt DF*// *Univ Virginia, Engn Phys Program, Charlottesville, Va 22901, USA

J Proteome Res 20043 (3) 621

Selective dissociation of non-covalent bonds in biological molecules by laser spray

Takamizawa A, Itoh $\mathrm{Y}$, Osawa R, Iwasaki N, Nishimura $\mathrm{Y}$, Akashi S* Hiraoka K// *Yokohama City Univ, Grad Sch Integrated Sci, 1-7-29 Suehiro cho, Tsurmi ku, Yokohama 230 0045, Japan

J Mass Spectrom 200439 (9) 1053

Electron capture dissociation Fourier transform ion cyclotron resonance mass spectrometry in the electron energy range $0-50 \mathrm{eV}$

Tsybin YO, Witt M, Baykut G, Hakansson P*//*Angstrom Lab, Div Ion Phys, Box 534, SE-75121 Uppsala, Sweden

Rapid Commun Mass Spectrom 200418 (14) 1607

Effect of sodium dodecyl sulfate micelles on peptide mass fingerprinting by matrix-assisted laser desorption/ionization mass spectrometry

Tummala R, Limbach PA*// *Univ Cincinatti, Dept Chem, Rieveschl Lab Mass Spectrom, POB 210172, Cincinatti, Oh 45221, USA

Rapid Commun Mass Spectrom 200418 (18) 2031

High-pressure ion trap mass spectrometry

Whitten WB, Reilly PTA, Ramsey JM// Oak Ridge Nat Lab, PO Box 2008, MS 6142, Oak Ridge, Tn 37831, USA

Rapid Commun Mass Spectrom 200418 (15) 1749

Use of polymer-modified MALDI-MS probes to improve analyses of protein digests and DNA

Xu YD, Bruening ML, Watson $\mathrm{JT}^{*} / / *$ Michigan State Univ, Dept Chem, East Lansing, Mi 48824, USA

Anal Chem 200476 (11) 3106

Mass spectrometry as an emerging tool for systems biology

Yates JR// Scripps Res Inst, Dept Cell Biol, La Jolla, Ca, USA

Biotechniques 200436 (6) 917

Quantitative measurement of fathead minnow vitellogenin by liquid chromatography combined with tandem mass spectrometry using a signature peptide of vitellogenin

Zhang FG, Bartels MJ, Brodeur JC, Woodburn KB// Dow Chem Co USA, Toxicol \& Environ Res \& Consulting, 1803 Bldg, Midland, Mi 48674, USA Environ Toxicol Chem 200423 (6) 1408

\section{GAS PHASE ION CHEMISTRY}

Obtaining thermochemical data by the extended kinetic method 
Bouchoux G, Sablier M, Berruyer-Penaud F// Ecole Polytech, Lab Mecanismes Reactionnels, UMR CNRS 7651, FR-91128 Palaiseau, France J Mass Spectrom 200439 (9) 986

Formation of molecular radical cations of aliphatic tripeptides from their complexes with $\mathrm{Cu}^{\mathrm{II}}(12$-crown-4)

Chu IK, Siu SO, Lam CNW, Chan JCY, Rodriquez CF// Univ Hong Kong, Dept Chem, Hong Kong, Peoples Rep China

Rapid Commun Mass Spectrom 200418 (16) 1798

Modeling of the gas-phase ion chemistry of protonated arginine

Csonska IP, Paizs B*, Suhai S// *Deutsches Krebsforschungszentrum, Dept Mol Biophys, Im Neuenheimer Feld 280, DE069120 Heidelberg, Germany

J Mass Spectrom 200439 (9) 1025

Accuracy of enthalpy and entropy determination using the kinetic method: Are we approaching a consensus?

Drahos L, Peltz C, Vekey K*// *Hungarian Acad Sci, Inst Structural Chem, Mass Spectrometry Dept, Pusztaszeri ut 59-67, HU-1025 Budapest, Hungary J Mass Spectrom 200439 (9) 1016

Systematic and random errors in ion affinities and activation entropies from the extended kinetic method

Ervin KM, Armentrout PB// Univ Nevada, Dept Chem \& Chem Phys Prog, Reno, Nv 89557, USA

J Mass Spectrom 200439 (9) 1004

Formation of iminium ions by fragmentation of $\mathrm{a}_{2}$ ions

Harrison AG, Young AB, Schnoelzer M, Paizs B// Univ Toronto, Dept Chem, 80 St George St, Toronto, Ontario, Canada M5S 3H6

Rapid Commun Mass Spectrom 200418 (14) 1635

Stereochemical preference in the loss of cyclohexene from ionized cyclohexyl phenyl ether (Letter)

Molenaar-Langeveld TA, Van der Burg LM, Peeters JWH, De Koster CG, Ingemann $\mathrm{S} * / / *$ Univ Amsterdam, Fac Sci, Swammerdam Inst Life Sci, Nieuwe Achtergracht 166, NL-1018 WV Amsterdam, The Netherlands J Mass Spectrom 200439 (9) 1075

Chiral recognition in solution and the gas phase. Experimental and theoretical studies of aromatic D- and L-amino acid-Cu(II)-chiragen complexes

Seymour JL, Turecek F*, Malkov AV, Kocovsky P// *Univ Washington, Dept Chem, PO Box 351700, Seattle, Wa 981195, USA

J Mass Spectrom 200439 (9) 1044

Entropy considerations in kinetic method experiments

Wesdemiotis C// Univ Akron, Dept Chem, Akron, Oh 44325, USA

J Mass Spectrom 200439 (9) 998

\section{BIOLOGY/BIOCHEMISTRY:}

\section{Amino acids, peptides \& proteins}

Analysis of substance $\mathrm{P}$ in rat brain by means of immunoaffinity capture and matrix-assisted laser desorption/ionization time-of-flight mass-spectrometry Babu CVS, Lee J, Lho DS, Yoo YS*// *Korea Inst Sci \& Technol, Bioanal \& Biotransformat Res Ctr, POB 131, Seoul 130 650, South Korea J Chromatogr B $2004 \mathbf{8 0 7}$ (2) 307

Absolute quantification of the model biomarker prostate-specific antigen in serum by LC-MS/MS using protein cleavage and isotope dilution mass spectrometry

Barnidge DR, Goodmanson MK, Klee GG, Muddiman DC*// *Mayo Clin, Coll Med, WM Keck FT-ICR Mass Spectrometry Lab, Mayo Proteomics Res Ctr, 200 1st St SW, Rochester, Mn 55905, USA

J Proteome Res 20043 (3) 644

Evaluation of a cleavable stable isotope labeled synthetic peptide for absolute protein quantification using LC-MS/MS

Barnidge DR, Hall GD, Stocker JL, Muddiman DC*//*Address as above

J Proteome Res 20043 (3) 658

Global analysis of the membrane subproteome of Pseudomonas aeruginosa using liquid chromatography-tandem mass spectrometry

Blonder J, Goshe MB, Xiao WZ, Camp DG, Wingerd M, Davis RW, Smith RD*// *Pacific NW Natl Lab, Environm Mol Sci Lab, POB 999, MSIN K8-98, Richland, Wa 99352, USA

J Proteome Res 20043 (3) 434

Co-populated conformational ensembles of $\beta_{2}$-microglobulin uncovered quantitatively by electrospray ionization mass spectrometry

Borysik AJH, Radford SE, Ashcroft AE*// *Univ Leeds, Sch Biochem \& Microbiol, Astbury Ctr Struct Mol Biol, Leeds LS2 9JT, England J Biol Chem 2004279 (26) 27069

Mapping of bovine skeletal muscle proteins using two-dimensional gel electrophoresis and mass spectrometry

Bouley J, Chambon C, Picard B*// *INRA, Lab Croissance \& Metab Muscle, Unite Rech Herbivores, FR-63122 St Genes Champanelle, France
Proteomics 20044 (6) 1811

Screening of sugar converting enzymes using quantitative MALDI-ToF mass spectrometry

Bungert D, Bastian S, Heckmann-Pohl DM, Giffhorn F, Heinzle E, Tholey A*// *Univ Saarland, DE-66123 Saarbrucken, Germany

Biotechnol Lett 200426 (13) 1025

Analysis of the sinusitis nasal lavage fluid proteome using capillary liquid chromatography interfaced to electrospray ionization-quadrupole time of flight-tandem mass spectrometry

Casado B, Pannell LK, Viglio S, Iadarola P, Baraniuk JN// Georgetown Univ, Div Rheumatol Allergy \& Immunol, Washington, DC 20007, USA

Electrophoresis 200425 (9) 1386

Characterization of the Salmonella typhimurium proteome by semi-automated two dimensional HPLC-mass spectrometry: Detection of proteins implicated in multiple antibiotic resistance

Coldham NG, Woodward MJ// Vet Labs Agcy, Dept Food \& Environ Safety, Addlestone KT15 3NB, England

J Proteome Res 20043 (3) 595

Unimod: Protein modifications for mass spectrometry

Creasy DM, Cottrell JS*// *Matrix Science Ltd, 8 Wyndham Pl, London W1H 1PP, England

Proteomics 20044 (6) 1534

Mass spectrometric identification of RNA binding proteins from dried EMSA gels

Dominique SR, Gruissem W, Baginsky S*// *Swiss Fed Inst Technol, ETH Zentrum, Inst Plant Sci, LFW E18, Universitatsstr 2, CH-8092 Zurich, Switzerland

J Proteome Res 20043 (3) 662

Identification and characterization of a new $\beta$-casein variant in goat milk by high-performance liquid chromatography with electrospray ionization mass spectrometry and matrix-assisted laser desorption/ionization mass spectrometry

Galliano F, Saletti R, Cunsolo V, Foti S*, Marletta D, Bordonaro S, D'Urso G// *Univ Catania, Dipt Sci Chim, Viale A Doria 6, IT-95125 Catania, Italy Rapid Commun Mass Spectrom 200418 (17) 1972

Expanded coverage of the human heart mitochondrial proteome using multidimensional liquid chromatography coupled with tandem mass spectrometry

Gaucher SP, Taylor SW, Fahy E, Zhang B, Warnock DE, Ghosh SS, Gibson BW*// *8001 Redwood Blvd, Novato, Ca 94945, USA

J Proteome Res 20043 (3) 495

A rapid method for analyzing recombinant protein inclusion bodies by mass spectrometry

Grimm R, Huber R, Neumeier T, Seidl A, Haslbeck M, Seibert FS*// *Hexal Biotech Forschungs GmbH, Keltenring 1\&3, DE-82041 Oberhaching, Germany

Anal Biochem 2004330 (1) 140

LC-MS/MS identification and yeast polymerase $\eta$ bypass of a novel $\gamma$-irradiation-induced intrastrand cross-linked lesion G[8-5]C

Gu CN, Wang YS*// *Univ Calif Riverside, Dept Chem, Riverside, Ca 92521, USA

Biochemistry 200443 (21) 6745

Low-molecular-weight human serum proteome using ultrafiltration, isoelectric focusing, and mass spectrometry

Harper RG, Workman SR, Schuetzner S, Timperman AT, Sutton JN*// *West Virginia Univ, Dept Chem, Protea Biosci Inc, Clark Hall, Morgantown, WV 26506, USA

Electrophoresis 200425 (9) 1299

Absolute quantification of proteins in solutions and in polyacrylamide gels by mass spectrometry

Havlis J, Shevchenko A*// *Max Planck Inst Mol Cell Biol \& Genet, Pfotenhauerstr 108, DE-01307 Dresden, Germany

Anal Chem 200476 (11) 3029

Investigation of non-covalent interactions between paramagnetic complexes and human serum albumin by electrospray mass spectrometry

Henrotte V, Laurent S, Gabelica V, Vander Elst L, Depauw E, Muller RN*// *Univ Mons-Hainaut, Dept Organic Chem, NMR Lab, 24 Ave du Champ de Mars, BE-7000 Mons, Belgium

Rapid Commun Mass Spectrom 200418 (17) 1919

Identification of phosphorylation sites in protein kinase A substrates using artificial neural networks and mass spectrometry

Hjerrild M, Stensballe A, Rasmussen TE, Kofoed CB, Blom N, SicheritzPonten T, Larsen MR, Brunak S, Jensen ON, Gammeltoft S// Glostrup Hosp, Dept Clin Biochem, Nordre Ringvej 57, DK-2600 Glostrup, Denmark J Proteome Res 20043 (3) 426

Development of an isotope dilution mass spectrometry assay for $\mathrm{HbA} 1 \mathrm{c}$ based on enzyme-cleaved peptide analysis 
Iguchi K, Nakanishi T, Miyazaki A, Shimizu A*, Ota A// *Osaka Med Coll, Clin Lab, 2-7 Daigakumachi, Takatsuki, Osaka 569 8686, Japan

J Chromatogr B 2004806 (1) 25

Proteome analysis in the bovine adrenal medulla using liquid chromatography with tandem mass spectrometry

Jalili PR, Dass C*// *Univ Memphis, Dept Chem, Campus Box 526060, Memphis, Tn 38152, USA

Rapid Commun Mass Spectrom 200418 (17) 1877

Phosphoproteome analysis of mouse liver using immobilized metal affinity purification and linear trap mass spectrometry

Jin WH, Dai J, Zhou H, Xia QC, Zou HF, Zeng R*// *Chinese Acad Sci, Shanghai Inst Biol Sci, Inst Biochem \& Cell Biol, Res Ctr Proteome Anal, 230 YueYang Rd, CN-200031 Shanghai, China

Rapid Commun Mass Spectrom 200418 (18) 2169

Proteome analysis of DNA damage-induced neuronal death using high throughput mass spectrometry

Johnson MD, Yu LR, Conrads TP, Kinoshita Y, Uo T, Matthews JD, Lee SW, Smith RD, Veenstra TD, Morrison RS*// *Univ Washington Sch Med, Dept Neurol Surg, Box 356470, 1959 NE Pacific St, Seattle, Wa 98195, USA

J Biol Chem 2004279 (25) 26685

Metabolic regulation analysis of icd-gene knockout Escherichia coli based on 2D electrophoresis with MALDI-TOF mass spectrometry and enzyme activity measurements

Kabir MM, Shimizu K*// *Kyushu Inst Technol, Dept Biochem Eng \& Sci, Fukuoka 820 8502, Japan

Appl Microbiol Biotechnol 200465 (1) 84

Proteomic analysis of Acinetobacter lwoffii K24 by 2-D gel electrophoresis and electrospray ionization quadrupole-time of flight mass spectrometry

Kim EA, Kim JY, Kim SJ, Park KR, Chung HJ, Leem SH, Kim SI*// *Korea Basic Sci Inst, Proteome Anal Team, 52 Yeoeun dong, Taejon 305 806, South Korea

J Microbiol Methods 200457 (3) 337

Correlations between air levels of hexahydrophthalic anhydride (HHPA) and HHPA-adducted albumin tryptic peptides in nasal lavage fluid from experimentally exposed volunteers

Kristiansson $\mathrm{MH}$, Lindh $\mathrm{CH}$, Jonsson BAG// Univ Hosp, Dept Occupat \& Environm Med, SE-22185 Lund, Sweden

Rapid Commun Mass Spectrom 200418 (14) 1592

Formation of $\mathrm{c}_{1}$ fragment ions in collision-induced dissociation of glutamine-containing peptide ions: A tip for de novo sequencing

Lee YJ, Lee YM*//*Univ Calif, Molec Structure Facil, One Shields Ave, Davis, Ca 95616, USA

Rapid Commun Mass Spectrom 200418 (18) 2069

Identification of membrane proteins differentially expressed in human papillomavirus type 16 E5-transfected human keratinocytes by nanoelectrospray ionization mass spectrometry

Leykauf K, Salek M, Schluter H, Lehmann WD, Alonso A*// *Deutsch Krebsforschungszentrum, Im Neuenheimer Feld 242, DE-69120 Heidelberg, Germany

J Gen Virol 200485 (6) 1427

Human serum proteins preseparated by electrophoresis or chromatography followed by tandem mass spectrometry

Marshall J, Jankowski A, Furesz S, Kireeva I, Barker L, Dombrovsky M, Zhu WM, Jacks K, Ingratta L, Bruin J, Kristensen E, Zhang R, Stanton E, Takashashi M, Jackowski G// Ryerson Univ, Fac Engn \& Appl Sci, Dept Chem \& Biol, Toronto, Ontario, Canada

J Proteome Res 20043 (3) 364

Substrate profiling of deubiquitin hydrolases with a positional scanning library and mass spectrometry

Mason DE, Ek J, Peters EC, Harris JL*//*Genomics Inst Novartis Res Fdn, 10675 John Jay Hopkins Dr, San Diego, Ca 92121, USA

Biochemistry 200443 (21) 6535

Identification of variant forms of the neuroendocrine peptide galanin

Norberg A, Griffiths WJ*, Hjelmqvist L, Jornvall H, Rokaeus A// *Univ London, Sch Pharm, Dept Pharmaceut \& Biol Chem, 29/39 Brunswick Sq, Bloomsbury, London WC1N 1AX, England

Rapid Commun Mass Spectrom 200418 (14) 1583

Method for differential detection and identification of components in protein mixtures analyzed by matrix-assisted laser desorption/ionization time-of-flight mass spectrometry

Ossipova E, Nord LI, Kenne L, Eriksson J*// *Swedish Univ Agr Sci, Dept Chem, POB 7015, SE-75007 Uppsala, Sweden Rapid Commun Mass Spectrom 200418 (18) 2053

Determination of $N$-acetyl-S-(N-methylcarbamoyl)cysteine (AMCC) in urine by high-performance liquid chromatography combined with mass spectrometry Perico A, Baglioni S, Bavazzano P// Lab Sanita Pubbl Azienda Sanitaria Firenze, Via San Salvi 12, IT-50135 Florence, Italy
Rapid Commun Mass Spectrom 200418 (16) 1865

Peptidomics of CNS-associated neurohemal systems of adult Drosophila melanogaster: A mass spectrometric survey of peptides from individual flies Predel R, Wegener C, Russell WK, Tichy SE, Russell DH, Nachman RJ// Saxon Acad Sci, Res Grp Jena, Erbertstr 1, DE-07743 Jena, Germany J Comp Neurol 2004474 (3) 379

Miniaturized proteomics and peptidomics using capillary liquid separation and high mass spectrometry

Ramstrom M, Bergquist J*// *Univ Uppsala, Dept Chem, Biomed Ctr, POB 599, SE-75124 Uppsala, Sweden

FEBS Lett 2004567 (1) 92

Rapid characterization of amyloid- $\beta$ side-chain oxidation by tandem mass spectrometry and the scoring algorithm for spectral analysis

Schiewe AJ, Margol L, Soreghan BA, Thomas SN, Yang AJ*// *Univ Sthn Calif, Dept Pharmaceut Sci, Los Angeles, Ca 90033, USA

Pharmaceut Res 200421 (7) 1094

Quantification of urinary 5-aminolevulinic acid by gas chromatography-mass spectrometry

Shinka T, Kuhara T// Kanazawa Med Univ, Med Res Inst, Div Human Genet, 1-1 Daigaku, Uchinada, Ishikawa 920 029, Japan

J Chromatogr B 2004806 (1) 19

Mass spectral study of Boc-carbo- $\beta^{3}$-peptides: Differentiation of two pairs of positional and diastereomeric isomers

Srikanth R, Reddy PN, Narsimha R, Srinivas R*, Sharma GVM, Reddy KR, Krishna PR// *Indian Inst Chem Technol, Natl Ctr Mass Spectrometry, IN-500007 Hyderabad, India

J Mass Spectrom 200439 (9) 1068

Phosphoric acid enhances the performance of Fe(III) affinity chromatography and matrix-assisted laser desorption/ionization tandem mass spectrometry for recovery, detection and sequencing of phosphopeptides

Stensballe A, Jensen ON*// *Univ Sthn Denmark, Dept Biochem \& Mol Biol, Protein Res Grp, Campusvej 55, DK-5230 Odense M, Denmark

Rapid Commun Mass Spectrom 200418 (15) 1721

Selective detection of glycopeptides on ion trap mass spectrometers

Sullivan B, Addona TA, Carr SA*// *Millennium Pharmaceut, Prot Sci \& Technol Dept, 45 Sidney St, Cambridge, Ma 02139, USA

Anal Chem 200476 (11) 3112

MALDI-TOF analysis of portal sera of pancreatic cancer patients: Identification of diabetogenic and antidiabetogenic peptides

Valerio A, Basso D, Fogar P, Falcon M, Greco E, Bassi C, Seraglia R, Abu-Hilal M, Navaglia F, Zambon CF, Gallo N, Falda A, Pedrazzoli S, Plebani $\mathrm{M} * / / *$ Univ Padua, Dept Lab Med, Via Giustiniani 2, IT-35128 Padua, Italy

Clin Chim Acta 2004343 (1-2) 119

Measurement of hepatic phenylalanine metabolism in children using the $\left[{ }^{13} \mathrm{C}\right]$-phenylalanine breath test and gas chromatography-mass spectrometry Wada M// Ichikawa Gen Hosp, Tokyo Dent Coll, Dept Pediat, 5-11-13 Sugano, Ichikawa, Chiba 272 8513, Japan J Chromatogr B 2004806 (1) 5

Proteomic patterns established with capillary electrophoresis and mass spectrometry for diagnostic purposes

Weissinger EM, Wittke S, Kaiser T, Haller H, Bartel S, Krebs R, Golovko I, Rupprecht HD, Haubitz M, Hecker H, Mischak H, Fliser D*//*Hannover Med Sch, Dept Nephrol, Carl Neuberg Str 1, DE-30625 Hannover, Germany Kidney Int $2004 \mathbf{6 5}$ (6) 2426

Glycopeptide analysis by matrix-assisted laser desorption/ionization tandem time-of-flight mass spectrometry reveals novel features of horseradish peroxidase glycosylation

Wuhrer W, Hokke CH, Deelder AM// Leiden Univ Med Ctr, Dept Parasitol, Ctr Infect Dis, PO Box 9600, NL-2300 RC Leiden, The Netherlands

Rapid Commun Mass Spectrom 200418 (15) 1741

Quantitative gas chromatography/mass spectrometry determination of Cmannosylation of tryptophan residues in glycoproteins

Zanetta JP, Pons A, Richet C, Huet G, Timmerman P, Leroy Y, Bohin A, Bohm JP, Trinel PA, Poulain D, Hofsteenge J// Univ Sci \& Tech Lille Flandres Artois, Unite Mixte Rech CNRS 8576, Batiment C9, FR-59655 Villeneuve d'Ascq, France Anal Biochem 2004329 (2) 199

Proteomic analysis of ductal carcinoma of the breast using laser capture microdissection, LC-MS, and ${ }^{16} \mathrm{O} /{ }^{18} \mathrm{O}$ isotopic labeling

Zang L, Toy DP, Hancock WS, Sgroi DC, Karger BL*// *Northeastern Univ, Dept Chem, Barnett Inst, 341 Mugar Bldg, 300 Huntington Ave, Boston, Ma 02115, USA

J Proteome Res 20043 (3) 604

Ultraviolet matrix-assisted laser desorption/ionization time-of-flight mass spectrometry of intact hemoglobin complex from whole human blood 
Zehl M, Allmaier G*//*Vienna Univ Technol, Inst Chem Technol \& Anal, Getreidemarkt 9/164-AC, AT-1060 Vienna, Austria

Rapid Commun Mass Spectrom 200418 (17) 1932

Proteomic analysis of SARS associated coronavirus using two-dimensional liquid chromatography mass spectrometry and one-dimensional sodium dodecyl sulfate-polyacrylamide gel electrophoresis followed by mass spectrometric analysis

Zeng R, Ruan HQ, Jiang XS, Zhou H, Shi L, Zhang L, Sheng QH, Tu QA, Xia QC, Wu JR// CAS, Shanghai Inst Biol Sci, Inst Biochem \& Cell Biol, Mol Cell Biol Lab, 320 YueYang Rd, CN-200031 Shanghai, Peoples Rep China

J Proteome Res 20043 (3) 549

Intact mass measurements for unequivocal identification of hydrophobic photosynthetic photosystems I and II antenna proteins

Zolla L, Rinalducci S, Timperio AM, Huber CG, Righetti PG// Univ Tuscia, Dept Environm Sci, Largo Univ, IT-01100 Viterbo, Italy

Electrophoresis 200425 (9) 1353

\section{Carbohydrates}

Kinetic measurements of phosphoglucomutase by direct analysis of glucose1-phosphate and glucose-6-phosphate using ion/molecule reactions and Fourier transform ion cyclotron resonance mass spectrometry

Gao H, Leary JA*// *Univ Calif Berkeley, Dept Chem, Berkeley, Ca 94720 , USA

Anal Biochem 2004329 (2) 269

Development and application of a rapid diagnostic method for invasive candidiasis by the detection of D-/L-arabinitol using gas chromatography/ mass spectrometry

Hui M, Cheung SW, Chin ML, Chu KC, Chan RCY, Cheng AFB// Chinese Univ Hong Kong, Prince of Wales Hosp, Dept Microbiol, Hong Kong, Peoples Rep China

Diagn Microbiol Infect Dis 200449 (2) 117

Site-specific $N$-glycosylation analysis: Matrix-assisted desorption/ionization quadrupole-quadrupole time-of-flight tandem mass spectral signatures for recognition and identification of glycopeptides

Krokhin O, Ens W, Standing KG, Wilkins J, Perreault H// Univ Manitoba, Manitoba Ctr Proteomics, Winnipeg, Manitoba, Canada

Rapid Commun Mass Spectrom 200418 (18) 2020

Determination of linkage position and anomeric configuration in Hex-Fuc disaccharides using electrospray ionization tandem mass spectrometry

Xue J, Song L, Khaja SD, Locke RD, West CM, Laine RA, Matta KL*// *Roswell Park Cancer Inst, Dept Cancer Biol, Elm \& Carlton St, Buffalo, NY 14263, USA

Rapid Commun Mass Spectrom 200418 (17) 1947

\section{Lipids}

Determination of phosphatidylcholine monohydroperoxides using quadrupole time-of-flight mass spectrometry

Adachi J, Yoshioka N, Funae R, Nushida H, Asano M, Ueno Y// Kobe Univ, Grad Sch Med, Dept Legal Med, 7 Kusunoki cho, Chuo ku, Kobe, Hyogo 650 0017, Japan

J Chromatogr B 2004806 (1) 41

Time-of-flight secondary ion mass spectrometry can replace histochemistry demonstration of fatty acids in the retina

Amemiya T, Tozu M, Ohashi Y// Nagasaki Univ, Grad Sch Biomed Sci, Dept Ophthalmol \& Visual Sci, Nagasaki 852 8501, Japan

Jpn J Ophthalmol 200448 (3) 287

Liquid chromatographic-mass spectrometric method to assess cytochrome P450-mediated metabolism of testosterone by rat everted gut sacs

Arellano C, Philibert C, Lacombe O, Woodley J, Houin G*//*UPTE, Fac Pharmaceut Sci, INRA-ENVT, Lab Cinet Xenobiot, UMR 181, 35 Chemin Maraichers, FR-31062 Toulouse, France

J Chromatogr B 2004807 (2) 263

Gas chromatography/mass spectrometry analysis of exhaled leukotrienes in asthmatic patients

Cap P, Chladek J, Pehal F, Maly M, Petru V, Barnes PJ, Montuschi P*// *Univ Sacred Heart, Fac Med, Dept Pharmacol, Largo F Vito 1, IT-00168 Rome, Italy

Thorax 200459 (6) 465

Mass spectrometric characterization of the oxidation of the fluorescent lipid peroxidation reporter molecule C11-BODIPY ${ }^{581 / 591}$

Drummen GPC, Gadella BM, Post JA, Brouwers JF*// *Univ Utrecht, Fac Med Vet, Dept Biochem \& Cell Biol, POB 80176, NL-3584 TD Utrecht, The Netherlands

Free Radic Biol Med 200436 (12) 1635
Off-line coupling of non-aqueous reversed-phase and silver ion high-performance liquid chromatography-mass spectrometry for the characterization of rice oil triacylglycerol positional isomers

Dugo P, Favoino O, Tranchida PQ, Dugo G, Mondello L// Univ Messina, Fac Sci, Dipt Chim Organ \& Biol, Salita Sperone 31, IT-98166 Messina, Italy J Chromatogr A 20041041 (1-2) 135

Evaluation of sphingolipids in vitreous bodies from a patient with Gaucher disease, using delayed extraction matrix-assisted laser desorption ionization time-of-flight mass spectrometry

Fujiwaki T, Yamaguchi S, Tasaka M, Takayanagi M, Isobe M, Taketomi T// Shimane Univ, Sch Med, Dept Pediat, 89-1 Enya cho, Izumo, Shimane 693 8501, Japan

J Chromatogr B 2004806 (1) 47

Gas chromatographic separation and mass spectrometric identification of mixtures of oxyphytosterol and oxycholesterol derivatives - Application to a phytosterol-enriched food

Grandgirard A, Martine L, Joffre C, Juaneda P, Berdeaux O// INRA, Unite Nutr Lipid, 17 rue Sully, BP 86510, FR-21065 Dijon, France

J Chromatogr A $2004 \mathbf{1 0 4 0}$ (2) 239

Simultaneous analysis of phospholipid in rabbit bronchoalveolar lavage fluid by liquid chromatography/mass spectrometry

Hayakawa J, Okabayashi Y// Shionogi \& Co Ltd, Shionogi Res Labs, 12-4 Sagisu 5 chome, Fukushima ku, Osaka 553 0002, Japan

J Pharm Biomed Anal 200435 (3) 583

Identification of monohydroxy progesterones produced by CYP106A2 using comparative HPLC and electrospray ionisation collision-induced dissociation mass spectrometry

Lisurek M, Kang MJ, Hartmann RW, Bernhardt R*// *Univ Saarland, FR 8.8 Biochemie, Postfach 151150, DE-66041 Saarbrucken, Germany

Biochem Biophys Res Commun 2004319 (2) 677

Determining the relative amounts of positional isomers in complex mixtures of triglycerides using reversed-phase high-performance liquid chromatographytandem mass spectrometry

Malone M, Evans JJ*// *Univ Massachusetts, Dept Chem, 100 Morrissey Blvd, Boston, Ma 02125, USA

Lipids 200439 (3) 273

Bacterial phospholipid molecular species analysis by ion-pair reversed-phase HPLC/ESI/MS

Mazzella N, Molinet J, Syakti AD, Dodi A, Doumenq P*, Artaud J, Bertrand JC// *Inst Fed Rech Pole Mediteranneen Sci Environm, Lab Chim Analyt Environm, Unite Mixte Rech 6171, 1122 Europole Arbois, BP 80, FR-13545 Aix-en-Provence 4, France

J Lipid Res 200445 (7) 1355

Quantification of linolenic acid isomers by gas chromatography-mass spectrometry and deconvolution of overlapping chromatographic peaks

Mjos SA// Norwegian Inst Fisheries \& Aquaculture Res, Kjerreidviken 16, NO-5141 Fyllingsdalen, Norway

Eur J Lipid Sci Technol 2004106 (5) 307

Negative electrospray ionization low-energy tandem mass spectrometry of hydroxylated fatty acids: A mechanistic study

Moe MK, Strom MB, Jensen E, Claeys M// Univ Tromso, Fac Med, Inst Pharm, Dept Med \& Bioanalyt Chem, NO-9037 Tromso, Norway

Rapid Commun Mass Spectrom 200418 (15) 1731

Vicinal hydroxylation of unsaturated fatty acids for structure characterization of intact neutral phospholipids by negative electrospray ionization tandem quadrupole mass spectrometry

Moe MK, Anderssen T, Strom MB, Jensen E// Univ Tromso, Inst Pharm, Dept Med \& Bioanal Chem, NO-9037 Tromso, Norway

Rapid Commun Mass Spectrom 200418 (18) 2121

LC/MS analysis and lipase modification of the sophorolipids produced by Rhodotorula bogoriensis

Nunez A, Ashby R, Foglia TA, Solaiman DKY// USDA/ARS, Fats Oils \& Anim Coprod Res Unit, Eastern Reg Res Ctr, 600 East Mermaid Lane, Wyndmoor, Pa 19038, USA

Biotechnol Lett 200426 (13) 1087

Bioimaging TOF-SIMS: Localization of cholesterol in rat kidney sections

Nygren H, Malmberg P, Kriegeskotte C, Arlinghaus HF// Univ Gothenburg, Dept Anat \& Cell Biol, POB 420, SE-40530 Gothenburg, Sweden

FEBS Lett 2004566 (1-3) 291

Determination of free fatty acids in chocolate by liquid chromatography with tandem mass spectrometry

Perret D, Gentili A, Marchese A, Sergi M, Caporossi L// Univ La Sapienza, Dipt Chim, Lab Chim Sicurezza, Piazzale Aldo Moro 5, PO Box 34, Posta 62, IT-00185 Rome, Italy

Rapid Commun Mass Spectrom 200418 (17) 1989

Mass spectrometric analysis of ceramide perturbations in brain and fibroblasts of mice and human patients with peroxisomal disorders 
Pettus BJ, Baes M, Busman M, Hannun YA, Van Veldhoven PP*// *KU Leuven, Campus Gasthuisberg, Afdeling Farmakologie, Postbus 601, Herestr, BE-3000 Leuven, Belgium

Rapid Commun Mass Spectrom 200418 (14) 1569

Formation of phosphatidic acid, ceramide, and deglyceride on radiolysis of lipids: Identification by MALDI-TOF mass spectrometry

Shadyro O, Yurkova I, Kisel M, Brede O, Arnhold J// Belarussian State Univ, Dept Chem, 4 Skaryna Ave, BY-220050 Minsk, Byelarus

Free Radic Biol Med 200436 (12) 1612

Testosterone metabolic clearance and production rates determined by stable isotope dilution/tandem mass spectrometry in normal men: Influence of ethnicity and age

Wang C, Catlin DH, Starcevic B, Leung A, DiStefano E, Lucas G, Hull L, Swerdloff RS// Harbor UCLA Med Ctr, Gen Clin Res Ctr, 1000 West Carson St, Torrance, Ca 90509, USA

J Clin Endocrinol Metab 200489 (6) 2936

Trace level quantification of deuterated $17 \beta$-estradiol and estrone in ovariectomized mouse plasma and brain using liquid chromatography/tandem mass spectrometry following dansylation reaction

Xia YQ, Chang SW, Patel S, Bakhtiar R, Karanam B, Evans DC// Merck Res Lab, Dept Drug Metabol, RY80L-109, PO Box 2000, Rahway, NJ 07065, USA

Rapid Commun Mass Spectrom 200418 (14) 1621

A gas chromatography/mass spectrometry assay to measure estradiol, catecholestradiols, and methoxyestradiols in plasma

Zacharia LC, Dubey RK, Jackson EK// Univ Pittsburgh Med Ctr, Dept Med, Ctr Clin Pharmacol, 623 Scaife Hall, 3550 Terrace St, Pittsburgh Pa 15261, USA

Steroids 200469 (4) 255

\section{Nucleic Acids}

Multiplexed hybridizations of positively charge-tagged peptide nucleic acids detected by matrix-assisted laser desorption/ionization time-of-flight mass spectrometry

Bauer O, Guerasimova A, Sauer S*, Thamm S, Steinfath M, Herwig R, Janitz M, Lehrach H, Radelof U// *Max-Planck-Inst Mol Genet, Dept Lehrach, Ihnestr 73, DE-14195 Berlin, Germany

Rapid Commun Mass Spectrom 200418 (16) 1821

Selective digestion and novel cleanup techniques for detection of benzo[a]pyrene diol epoxide-DNA adducts by capillary electrophoresis/mass spectrometry

Gennaro LA, Vadhanam M, Gupta RC, Vouros P*// *Northeastern Univ, Dept Chem \& Chem Biol, Barnett Inst, Boston, Ma 02115, USA

Rapid Commun Mass Spectrom 200418 (14) 1541

High-performance liquid chromatography-mass selective detection assay for adenine released from a synthetic RNA substrate by ricin A chain

Hines HB, Brueggemann EE, Hale ML// USA, Res Inst Infect Dis, Toxinol \& Aerobiol Div, Fort Detrick, Md 21702, USA

Anal Biochem 2004330 (1) 119

A high-throughput and sensitive methodology for the quantification of urinary 8-hydroxy-2'-deoxyguanosine: Measurement with gas chromatography-mass spectrometry after single solid-phase extraction

Lin HS, Jenner AM, Ong CN, Huang SH, Whiteman M, Halliwell B*// *Natl Univ Singapore, Fac Med, Dept Biochem, MD 7, Med Dr, SG-117597 Singapore, Singapore

Biochem J 2004380 (2) 541

A computational and experimental study of cation affinity $\left(\mathrm{Na}^{+}\right)$of nucleobases and modified nucleobases by electrospray ionization ion trap mass spectrometry

Rochut S, Pepe C*, Paumard JP, Tabet JC// *Univ Pierre \& Marie Curie, Lab Dynamique Interact \& React, UMR 7075, Paris, France

Rapid Commun Mass Spectrom 200418 (15) 1686

Implementation of data-dependent acquisitions in the study of melphalan DNA adducts by miniaturized liquid chromatography coupled to electrospray tandem mass spectrometry

Van den Driessche B, Van Dongen W, Lemiere F, Esmans EL*// *Univ Antwerp, Nucleoside Res \& Mass Spectrom Unit, Groenenborgerlaan 171, BE-2020 Antwerp, Belgium

Rapid Commun Mass Spectrom 200418 (17) 2001

Liquid chromatographic analysis of nucleosides and their mono-, di- and triphosphates using porous graphitic carbon stationary phase coupled with electrospray mass spectrometry

Xing J, Apedo A, Tymiak A, Zhao N// Bristol-Myers Squibb Pharm Res Inst, Pharm Candidate Optimization, Princeton, NJ 08543, USA

Rapid Commun Mass Spectrom 200418 (14) 1599

\section{PHARMACOLOGY/TOXICOLOGY}

Determination of benzoyl peroxide and benzoic acid in wheat flour by high-performance liquid chromatography and its identification by high-performance liquid chromatography-mass spectrometry

Abe-Onishi Y, Yomota C*, Sugimoto N, Kubota H, Tanamoto K// *Natl Inst Hlth Sci, Div Food Addit, 1-18-1 Kamiyoga, Setagaya ku, Tokyo 158 8501, Japan

J Chromatogr A 20041040 (2) 209

Characterization studies and cytotoxicity assays of $\mathrm{Pt}(\mathrm{II})$ and $\mathrm{Pd}(\mathrm{II})$ dithiocarbamate complexes by means of FT-IR, NMR spectroscopy and mass spectrometry

Alverdi V, Giovagnini L, Marzano C, Seraglia R, Bettio F, Sitran S, Graziani R, Fregona D*// *Univ Padua, Dept Inorgan Metallorgan \& Analyt Chem, Via Loredan 4, IT-35131 Padua, Italy

J Inorg Biochem 200498 (6) 1117

Analysis of anabolic steroids by partial filling micellar electrokinetic capillary chromatography and electrospray mass spectrometry

Amundsen LK, Kokkonen JT, Rovio S, Siren H*// *VTT Proc, POB 1602, FI-02044 Espoo, Finland

J Chromatogr A 20041040 (1) 123

Identification of diphenhydramine metabolites in human urine by capillary electrophoresis-ion trap-mass spectrometry

Baldacci A, Prost F, Thormann $\mathrm{W} * / / *$ Univ Bern, Dept Clin Pharmacol, Murtenstr 35, CH-3010 Bern, Switzerland

Electrophoresis 200425 (10-11) 1607

Simultaneous determination of $t, t$-muconic, $S$-phenylmercapturic and $S$-benzylmercapturic acids in urine by a rapid and sensitive liquid chromatography/electrospray tandem mass spectrometry method

Barbieri A, Sabatini L, Accorsi A, Roda A, Violante FS// Univ Bologna, Serv Sicurezza Igiene Med Lavoro, Via Palagi 9, IT-40138 Bologna, Italy Rapid Commun Mass Spectrom 200418 (17) 1983

Simple and rapid liquid chromatography-tandem mass spectrometry confirmatory assay for determining amoxicillin and ampicillin in bovine tissues and milk

Bogialli S, Capitolino V, Curini R, Di Corcia A*, Nazzari M, Sergi M// *Univ Roma La Sapienza, Dipt Chim, Piazza Aldo Moro 5, IT-00185 Rome, Italy

J Agric Food Chem 200452 (11) 3286

Rapid determination of chloramphenicol and its glucuronide in food products by liquid chromatography-electrospray negative ionization tandem mass spectrometry

Bogusz MJ, Hassan H, Al-Enazi E, Ibrahim Z, Al-Tufail M// King Faisal Specialist Hospital \& Research Ctr, POB 3354, SA-11211 Riyadh, Saudi Arabia

J Chromatogr B 2004807 (2) 343

Analysis of heterocyclic aromatic amines in foods by gas chromatographymass spectrometry as their tert-butyldimethylsilyl derivatives

Casal S, Mendes E, Fernandes JO, Oliveira MBPP, Ferrreira MA// Univ Porto, Fac Farm, Serv Bromatol, REQUIMTE, Rua Anibal Cunha 164, PT-4099-030 Oporto, Portugal

J Chromatogr A 20041040 (1) 105

Liquid chromatography-tandem mass spectrometry method for the determination of tranexamic acid in human plasma

Chang Q, Yin OQP*, Chow MSS//*Chinese Univ Hong Kong, Fac Med, Sch Pharm, Shatin, Hong Kong, Peoples Rep China

J Chromatogr B 2004805 (2) 275

Separation and identification of compounds in Rhizoma Chuanxiong by comprehensive two-dimensional liquid chromatography coupled to mass spectrometry

Chen XG, Kong L, Su XY, Fu HJ, Ni JY, Zhao RH, Zou HF*// *Chinese Acad Sci, Dalian Inst Chem Phys, Natl Chromatog R\&A Ctr, 161 Zhongshan Rd, CN-116011 Dalian, Peoples Rep China J Chromatogr A 20041040 (2) 169

Simultaneous determination of chlorpheniramine and pseudoephedrine in human plasma by liquid chromatography-tandem mass spectrometry

Chen XY, Zhang Y, Zhong DF*// *Shenyang Pharmaceut Univ, Lab Drug Metab \& Pharmacokinet, 103 Wenhua Rd, CN-110016 Shenyang, Peoples Rep China

Biomed Chromatogr 200418 (4) 248

Analysis of strychnine from detoxified Strychno nux-vomica seeds using liquid chromatography-electrospray mass spectrometry

Choi YH, Sohn YM, Kim CY, Oh KY, Kim J*// *Seoul Natl Univ, Coll Pharm, Seoul 151 742, South Korea

J Ethnopharmacol 200493 (1) 109

Determination of a novel substance $\mathrm{P}$ inhibitor in human plasma by highperformance liquid chromatography with atmospheric pressure chemical ion- 
ization mass spectrometric detection using single and triple quadrupole detectors

Constanzer ML, Chavez-Eng CM, Dru J, Kline WF, Matuszewski BK// Merck Res Labs, Sunneytown Pike, WP75A-303, West Point, Pa 19486,

USA

J Chromatogr B 2004807 (2) 243

Identification of iso- and $n$-propylphosphonates using liquid chromatography-tandem mass spectrometry and gas chromatography-Fourier transform infrared spectroscopy

Cooper DB, Read RW, Timperley CM, Williams NH*, Black RM// *Defence Sci \& Technol Lab, Salisbury SP4 0JQ, England

J Chromatogr A 20041040 (1) 83

Surface-activated chemical ionization ion trap mass spectrometry in the analysis of amphetamines in diluted urine samples

Cristoni S, Bernardi LR, Gerthoux P, Gonella E, Mocarelli P// Univ Milano,

Via Fratelli Cervi 93, IT-20090 Segrate, Milan, Italy

Rapid Commun Mass Spectrom 200418 (16) 1847

Quantitation of drug metabolites in the absence of pure metabolite standards by high-performance liquid chromatography coupled with a chemiluminescence nitrogen detector and mass spectrometer

Deng Y, Wu JT, Zhang H, Olah TV// Bristol-Myers Squibb Co, PO Box 4000, Princeton, NJ 08543, USA

Rapid Commun Mass Spectrom 200418 (15) 1681

Rapid and sensitive liquid chromatography-electrospray ionization-mass spectrometry method for the determination of eperisone in human plasma: Method and clinical applications

Ding L, Wei X, Zhang SQ, Sheng JP, Zhang YD// China Pharmaceut Univ, Dept Pharmaceut Anal, 24 Tongjiaxiang, CN-210009 Nanjing, Peoples Rep China

J Chromatogr Sci 200442 (5) 254

Adducts of N-terminal valines in hemoglobin with isoprene diepoxide, a metabolite of isoprene

Fred A, Cantillana T, Henderson AP, Golding BT, Tornqvist M*// *Stockholm Univ, Dept Environ Chem, SE-10691 Stockholm, Sweden

Rapid Commun Mass Spectrom 200418 (18) 2177

Determination of amphetamine in rat brain by in vivo microdialysis and nonpairing liquid chromatography with electrospray tandem mass spectrometry Fuh MR, Huang CH, Wu TY, Lin SL, Pan WHT// Soochow Univ, Dept Chem, Taipei, Taiwan

Rapid Commun Mass Spectrom 200418 (15) 1711

A computer program for automated data evaluation to support in vitro higher-throughput screening for drug metabolism and pharmacokinetics attributes

Fung EN, Chu I, Nomier AA*// *Schering-Plough Res Inst, Dept Drug Metab \& Pharmacokinet, MS 2880, 2015 Galloping Hill Rd, Kenilworth, NJ 07033, USA

Rapid Commun Mass Spectrom 200418 (18) 2046

Liquid chromatographic-mass spectrometric determination of endogenous $\gamma$ hydroxybutyrate concentrations in rat brain regions and plasma

Fung HL, Haas E, Raybon J, Xu J, Fung SM// SUNY Buffalo, Sch Pharm \& Pharmaceut Sci, Dept Pharmaceut Sci, Buffalo, NY 14260, USA

J Chromatogr B 2004807 (2) 287

Cell-based screen of HMG-CoA reductase inhibitors and expression regulators using LC-MS

Gerber R, Ryan JD, Clark DS*// *Univ Calif Berkeley, Dept Chem Engn, 201 Gilman Hall, Berkeley, Ca 94720, USA

Anal Biochem 2004329 (1) 28

Validated semiquantitative/quantitative screening of 51 drugs in whole blood as silylated derivatives by gas chromatography-selected ion monitoring mass spectrometry and gas chromatography electron capture detection

Gunnar T, Mykkanen S, Ariniemi K, Lillsunde P// Nat Publ Hlth Inst, Lab Substance Abuse, Mannerheimintie 166, FI-00300 Helsinki, Finland

J Chromatogr B 2004806 (2) 205

Analyzing of the volatile chemical constituents in Artemisia Capillaris Herba by GC-MS and correlative chemometric resolution methods

Guo FQ, Liang YZ*, Xu CH, Li XN, Huang LF//*Cent Sth Univ, Coll Chem \& Chem Engn, Res Ctr Modernizat Chinese Herbal Med, CN-410083 Changsha, Peoples Rep China

J Pharm Biomed Anal 200435 (3) 469

Development of a liquid chromatography-tandem mass spectrometric method for the determination of methamphetamine and amphetamine using small volumes of rat serum

Hendrickson HP, Milesi-Halle A, Laurenzana EM, Owens SM// Univ Arkansas Med Sci, Dept Pharmacol \& Toxicol, 4301 West Markham St, Little Rock, Ar 72205, USA

J Chromatogr B 2004806 (2) 81
Quantification of $1, N^{6}$-etheno-2'-deoxyadenosine in human urine by column-switching LC/APCI-MS/MS

Hillestrom PR, Hoberg AM, Weimann A, Poulsen HE*// *Rigshosp, Dept Clin Pharmacol, Q-7642, Tagensvej 20, DK-2200 Copenhagen N, Denmark Free Radic Biol Med 200436 (11) 1383

Quantification of the $N$-desmethyl metabolite of rosuvastatin in human plasma by automated SPE followed by HPLC with tandem MS detection

Hull CK, Martin PD, Warwick MJ, Thomas E// Heriot Watt Univ, Quintiles Scotland Ltd, Res Ave Sth, Res Pk, Edinburgh EH14 4AP, Scotland

J Pharm Biomed Anal 200435 (3) 609

Determination of chloralose residues in animal tissues by liquid chromatography-electrospray ionisation tandem mass spectrometry

Hunter K, Taylor MJ, Sharp EA, Melton LM, Le Bouhellec S// SASA, East Craigs, 82 Craigs Rd, Edinburgh EH12 8NJ, Scotland

J Chromatogr B 2004805 (2) 303

Application of liquid chromatography-tandem mass spectrometry for the determination of opioidmimetics in the brain dialysates from rats treated with opioidmimetics intraperitoneally

Igarashi K, Murabayashi Y, Hotta K, Kitamura Y, Kasuya F, Shiotani K, Li TY, Miyazaki A, Tsuda Y, Okada Y, Fukushima S// Kobe Gakuin Univ, Fac Pharmaceut Sci, Dept Analyt Toxicol, Ikawadani cho, Nishi ku, Kobe, Hyogo 6512180 , Japan

J Chromatogr B 2004806 (1) 53

Simultaneous determination of barbiturates in human biological fluids by direct immersion solid-phase microextraction and gas chromatography-mass spectrometry

Iwai M, Hattori $\mathrm{H}$, Arinobu T, Ishii A, Kumazawa T, Noguchi $\mathrm{H}$, Noguchi $\mathrm{H}$, Suzuki O, Seno H// Aichi Med Univ, Sch Med, Dept Legal Med, Nagakute, Aichi 480 1195, Japan

J Chromatogr B 2004806 (1) 65

Liquid chromatography-mass spectrometric analysis of compound $\mathrm{K}$, a ginseng saponin metabolite, in rat plasma

Ji HY, Lee HW, Kim HH, Kim HK, Kim YC, Sohn DH, Kim JB, Lee HS*// *Wonkwang Univ, Coll Pharm \& Phytofermentat Res Ctr, Drug Metab \& Bioanal Lab, Iksan 570 749, South Korea Anal Lett 200437 (7) 1307

Simultaneous quantitation of dexmedetomidine and glucuronide metabolites (G-Dex-1 and G-Dex-2) in human plasma utilizing liquid chromatography with tandem mass spectrometric detection

Ji QC, Zhou JY, Gonzales RJ, Gage EM, El-Shourbagy TA// Abbott Labs, Dept Drug Anal, Abbott Park, Il 60064, USA

Rapid Commun Mass Spectrom 200418 (15) 1753

Accurate assignment of ethanol origin in postmortem urine: Liquid chromatographic-mass spectrometric determination of serotonin metabolites Johnson RD, Lewis RJ, Canfield DV, Blank CL// FAA, Civil Aerosp Med Inst, Analyt Toxicol \& Accident Res Lab, Oklahoma City, Ok 73169, USA J Chromatogr B 2004805 (2) 223

Determination of benidipine in human plasma using liquid chromatographytandem mass spectrometry

Kang WK, Yun HY, Liu KH, Kwon KI, Shin JG*// *Inje Univ, Coll Med, Dept Pharmacol, Pusan, South Korea

J Chromatogr B 2004805 (2) 311

Quantification of mephenytoin and its metabolites 4'-hydroxymephenytoin and nirvanol in human urine using a simple sample processing method

Klaasen T, Kasel D, Harlfinger S, Fuhr U// Univ Cologne, Dept Pharmacol, Clin Pharmacol, Gleueler Str 24, DE-50931 Koln, Germany

Rapid Commun Mass Spectrom 200418 (15) 1675

Simultaneous determination of four immunosuppressants by means of high speed and robust on-line solid phase extraction-high performance liquid chromatography-tandem mass spectrometry

Koal T, Deters M, Casetta B, Kaever V// Hannover Med Sch, Inst Pharmacol, Carl Neuberg Str 1, DE-30625 Hannover, Germany

J Chromatogr B 2004805 (2) 215

Detection and determination of reticuline and $N$-methylcoculaurine in the Annonaceae family using liquid chromatography-tandem mass spectrometry Kotake Y, Okuda K, Kamizono M, Matsumoto N, Tanahashi T, Hara H, Caparros-Lefebvre D, Ohta S// Hiroshima Univ, Grad Sch Biomed Sci, 1-2-3 Kasumi, Minami ku, Hiroshima 734 8551, Japan

J Chromatogr B 2004806 (1) 75

Determination of bisphosphonate by ion chromatography-inductively coupled mass spectrometry

Kovacevic M, Gartner A, Novic M*// *Natl Inst Chem, POB 660, SI-1000 Ljubljana, Slovenia

J Chromatogr A 20041039 (1-2) 77

A study of the stability of tri(glucosyloxyphenyl)chlorin, a sensitizer for photodynamic therapy, in human colon tumoural cells: A liquid chromatogra- 
phy and MALDI-TOF mass spectrometry analysis

Laville I, Pigaglio S, Blais JC, Loock B, Maillard P, Grierson DS, Blais J*// *Univ Paris 06, LPBC, UMR CNRS 7033, 4 Pl Jussieu, Case 138, FR-75252 Paris 05, France

Bioorg Med Chem Lett 200412 (13) 3673

Synthesis and identification of two potential oxidation degradants of oxymetazoline

Leader IP, Halket J, Finch P// Royal Holloway, Egham TW20 0EX, England Rapid Commun Mass Spectrom 200418 (14) 1645

Metabolism of 1-\{3-[3-(4-cyanobenzyl)-3H-imidazol-4-yl]-propyl\}-3-(6-methoxypyridin-3-yl)-1-(2-trifluoromethylbenzyl)thiourea (YH3945), a novel anticancer drug, in rats using the ${ }^{14} \mathrm{C}$-labeled compound

Lee J, La S, Ahn BR, Jeong TC, Kim DH*// *Korea Inst Sci \& Technol, Bioanal \& Biotransformat Res Ctr, PO Box 131, Chungryang, Seoul 130-650, South Korea

Rapid Commun Mass Spectrom 200418 (17) 1901

In vitro and in vivo metabolism of pyronaridine characterized by low-energy collision-induced dissociation mass spectrometry with electrospray ionization Lee J, Son J, Chung SJ, Lee ES, Kim DH*//*Address as above

J Mass Spectrom 200439 (9) 1036

A metabonomic investigation of the biochemical effects of mercuric chloride in the rat using ${ }^{1} \mathrm{H}$ NMR and HPLC-TOP/MS: Time dependant changes in the urinary profile of endogenous metabolites as a result of nephrotoxicity

Lenz EM, Bright J, Knight R, Wilson ID*, Major H// *AstraZeneca Pharmaceut, Dept Drug Metab \& Pharmacokinet, Alderley Pk, Macclesfield SK10 4TG, England

Analyst 2004129 (6) 535

Cyclosporin A-induced changes in endogenous metabolites in rat urine: A metabonomic investigation using high field ${ }^{1} \mathrm{H}$ NMR spectroscopy, HPLCTOF/MS and chemometrics

Lenz EM, Bright J, Knight R, Wilson ID, Major H// AstraZeneca Pharmaceut, Dept Drug Metab, Alderley Pk, Macclesfield SK10 4TG, England

J Pharm Biomed Anal 200435 (3) 599

Determination of midazolam in human plasma by liquid chromatography with mass-spectrometric detection

Lepper ER, Hicks JK, Verweij J, Zhai SP, Figg WD*, Sparreboom A// *NCI, Canc Res Ctr, Clin Pharmacol Res Core, 9000 Rockville Pike, Bldg 10, Room 5A01, Bethesda, Md 20892, USA

J Chromatogr B 2004806 (2) 305

Simultaneous selected reaction monitoring, MS/MS and $\mathrm{MS}^{3}$ quantitation for the analysis of pharmaceutical compounds in human plasma using chip-based infusion

Leuthold LA, Grivet C, Allen M, Baumert M, Hopfgartner G*// *Univ Geneva, Sch Pharm, Lab Pharmaceut Analyt Chem Life Sci Mass Spectrom, 20 Blvd d'Yvoy, CH-1211 Geneva, Switzerland

Rapid Commun Mass Spectrom 200418 (17) 1995

Determination of cocaine, its metabolites, pyrolysis products, and ethanol adducts in postmortem fluids and tissues using Zymark ${ }^{\circledR}$ automated solid- phase extraction and gas chromatography-mass spectrometry

Lewis RJ, Johnson RD, Angier MK, Ritter RM// FAA, Civil Aerosp Med Inst, Toxicol \& Accident Res Lab, AAM-610, POB 25082, Oklahoma City, Ok 73125, USA

J Chromatogr B 2004806 (2) 141

Method development and validation for quantitative determination of methadone enantiomers in human plasma by liquid chromatography/tandem mass spectrometry

Liang HR, Foltz RL, Meng M, Bennett P// NWT Inc, Tandem Labs, 1121 East 3900 Sth, Salt Lake City, Ut 84124, USA

J Chromatogr B 2004806 (2) 191

Single-drop microextraction and gas chromatography/mass spectrometric determination of anisaldehyde isomers in human urine and blood serum

Liu BM, Malik P, Wu HF*// *Tamkang Univ, Dept Chem, Tamsui 251, Taiwan

Rapid Commun Mass Spectrom 200418 (18) 2059

Separation and determination of dexamethasone sodium phosphate in cochlear perilymph fluid by liquid chromatography with ultraviolet monitoring and electrospray ionization mass spectrometry characterization

Liu HX, Chen XL, Zhang SS, Qu LB, Zhao WF, Liu HJ, Dong MM// Zhengzhou Univ, Dept Chem, Key Lab Chem Biol \& Organ Chem Henan, 75 Daxue Rd, CN-450052 Zhengzhou, Peoples Rep China

J Chromatogr B 2004805 (2) 255

Determination of ochratoxin A in meat products by high-performance liquid chromatography coupled to electrospray ionisation sequential mass spectrometry

Losito I, Monaci L, Palmisano F*, Tantillo G// *Univ Bari, Dipt Chim, Via E Orabona 4, IT-70126 Bari, Italy

Rapid Commun Mass Spectrom 200418 (17) 1965
Determination of donepezil hydrochloride (E2020) in plasma by liquid chromatography-mass spectrometry and its application to pharmacokinetic studies in healthy, young, Chinese subjects

Lu YH, Wen HM, Wei W, Chi YM, Zhang ZX*// *China Pharmaceut Univ, Dept Pharmaceut Anal, CN-210009 Nanjing, Peoples Rep China

J Chromatogr Sci 200442 (5) 234

Determination of morphine, morphine-3-glucuronide and morphine-6glucuronide in monkey and dog plasma by high-performance liquid chromatography-electrospray ionization tandem mass spectrometry

Mabuchi M, Takatsuka S, Matsuoka M, Tagawa K// Tanabe Seiyaku Co Ltd, Analyt Dev Lab, 16-89 Kashima 3-chome, Yodogawa ku, Osaka 532 8505, Japan

J Pharm Biomed Anal 200435 (3) 563

Chemical screening by mass spectrometry to identify inhibitors of anthrax lethal factor

Min DH, Tang WJ, Mrksich M*// *Univ Chicago, Dept Chem, Inst Biophys Dynam, 5735 Sth Ellis Ave, Chicago, Il 60637, USA

Nat Biotechnol 200422 (6) 717

Enhanced method performance due to a shorter chromatographic run-time in a liquid chromatography-tandem mass spectrometry assay for paclitaxel Mortier KA, Verstraete AG, Zhang GF, Lambert WE*// *State Univ Ghent, Toxicol Lab, Harelbekestr 72, BE-9000 Ghent, Belgium

J Chromatogr A 20041041 (1-2) 235

Determination of halofuginone in eggs by liquid chromatography/tandem mass spectrometry after cleanup with immunoaffinity chromatography Mortier L, Daeseleire E, Huet AC, Delahaut P, Van Peteghem C// Ministry Flemish Commun, Agr Res Ctr Ghent, Dept Anim Prod Qual \& Transformation Technol, Brusselsesteenweg 370, BE-9090 Melle, Belgium

Rapid Commun Mass Spectrom 200418 (16) 1817

Matrix-assisted laser desorption/ionization time-of-flight mass spectrometry for monitoring and optimization of site specific PEGylation of ricin A-chain (Letter)

Na DH, Youn Y, Lee KC*// *SungKyunKwan Univ, Coll Pharm, Drug Target Lab, 300 Chonchon-dong, Jangun-ku, Suwon City 440 746, Korea Rapid Commun Mass Spectrom 200418 (18) 2185

Determination of neomycin by LC-tandem mass spectrometry using hydrophilic interaction chromatography

Oertel R, Renner U, Kirch W// Tech Univ Dresden, Med Fac Carl Gustav Carus, Inst Clin Pharmacol, Fiedlerstr 27, DE-01307 Dresden, Germany J Pharm Biomed Anal 200435 (3) 633

Detection of griseofulvin in a marine strain of Penicillium waksmanii by ion trap mass spectrometry

Petit KE, Mondeguer F, Roquebert MF, Biard JF, Pouchus YF*// *Univ Nantes, Fac Pharm, ISOMer, SMAB, BP 53 508, FR-44035 Nantes 01, France

J Microbiol Methods 200458 (1) 59

Development and validation of a gas chromatography-mass spectrometry method for the simultaneous determination of buprenorphine, flunitrazepam and their metabolites in rat plasma: Application to the pharmacokinetic study

Pirnay S, Bouchonnet S, Herve F, Libong D, Milan N, d'Athis P, Baud F, Ricordel I// Lab Toxicol Prefecture Police, 2 Pl Mazas, FR-75012 Paris, France

J Chromatogr B 2004807 (2) 335

Identification of puerarin and its metabolites in rats by liquid chromatography-tandem mass spectrometry

Prasain JK, Jones K, Brissie N, Moore R, Wyss JM, Barnes S// Univ Alabama, Dept Pharmacol \& Toxicol, Birmingham, Al 35294, USA J Agric Food Chem 200452 (12) 3708

Quantification of acarbose in human plasma by liquid chromatographyelectrospray tandem mass spectrometry

Raut BB, Kolte BL, Deo AA, Bagool MA, Shinde DB*//*Dr Babasaheb Ambedkar Marathwada Univ, Dept Chem Technol, IN-431004 Aurangabad, Maharashtra, India

J Liq Chromatogr Relat Technol 200427 (11) 1759

Quantitative determination of a novel insulin sensitizer and its parahydroxylated metabolite in human plasma by LC-MS/MS

Riffel KA, Polinko MA, Song HC, Rippley RK, Lo MW// Merck Res Labs, Dept Drug Metab, WP75A-303, West Point, Pa 19486, USA

J Pharm Biomed Anal 200435 (3) 523

Headspace solid-phase microextraction and capillary gas chromatographicmass spectrometric determination of rivastigmine in canine plasma samples Sha YF, Deng CH, Liu Z, Huang TM, Yang B, Duan GL*// *Fudan Univ, Dept Pharm, CN-200032 Shanghai, Peoples Rep China J Chromatogr B 2004806 (2) 271

Trace detection of glycolic acid by electrophore labeling gas chromatography-electron capture mass spectrometry 
Shao G, Giese RW*// *Northeastern Univ, Bouve Coll Pharm \& Hlth Professions, Dept Pharmaceut Sci, Boston, Ma 02115, USA

Anal Chem 200476 (11) 3049

Analysis of neutral drugs in human plasma by fluoride attachment in liquid chromatography/negative ion electrospray tandem mass spectrometry Sheen JF, Her GR*// *Nat Taiwan Univ, Dept Chem, Taipei, Taiwan Rapid Commun Mass Spectrom 200418 (17) 1911

Simultaneous determination of MK-0767 and seven metabolites in rat urine using liquid chromatography/tandem mass spectrometry

Shen Z, Kochansky C, Bakhtair R, Franklin RB, Vincent SH// Merck Res Lab, Mail Stop RY80L-109, POB 2000, Rahway, NJ 07065, USA

Rapid Commun Mass Spectrom 200418 (18) 2113

Mechanism of inactivation of $\beta$-lactamases by novel 6-methylidene penems elucidated using electrospray ionization mass spectrometry

Tabei K, Feng XD, Venkatesan AM, Abe T, Hideki U, Mansour TS, Siegel MM*// *Wyeth Ayerst Res, Chem Technol Chem \& Screening Sci, 401 Nth Middletown Rd, Bldg 222, Room 1043, Pearl River, NY 10965, USA J Med Chem 200447 (14) 3674

Monitoring of urinary acrolein concentration in patients receiving cyclophosphamide and ifosphamide

Takamoto S, Sakura N, Namera A, Yashiki M// Hiroshima Univ, Grad Sch Biomed Sci, Dept Pediat, 1-2-3 Kasumi, Minami ku, Hiroshima 734 8551, Japan

J Chromatogr B 2004806 (1) 59

Sensitive and selective liquid chromatography-electrospray ionization tandem mass spectrometry analysis of hydrochlorothiazide in rat plasma

Takubo T, Okada H, Ishii M, Hara K, Ishii Y// Banyu Pharmaceut Co Ltd, Tsukuba Res Inst, Drug Metab, 3 Okubo, Tsukuba, Ibaraki 300 2611, Japan J Chromatogr B 2004806 (2) 199

Electrospray ionization mass spectrometric characterization and quantitation of xanthine derivatives using isotopically labelled analogues: An application for equine doping control analysis

Thevis M, Opfermann G, Krug O, Schanzer W// German Sport Univ Cologne, Inst Biochem, Carl-Diem Weg 6, Cologne, Germany

Rapid Commun Mass Spectrom 200418 (14) 1553

Simultaneous quantification of beclomethasone dipropionate and its metabolite, beclomethasone 17-monopropionate in rat and human plasma and different rat tissue by liquid chromatography-positive electrospray ionization tandem mass spectrometry

Wang YN, Hochhaus G*// *Univ Florida, Coll Pharm, Dept Pharmaceut, POB 100494, Gainesville, Fl 32610, USA

J Chromatogr B 2004805 (2) 203

In-vitro metabolism of isotetrandrine, a bisbenzylisoquinoline alkaloid, in rat hepatic S9 fraction by high-performance liquid chromatography-atmospheric pressure ionization mass spectrometry

Wu WN, McKown LA, Gopaul VS*// *Johnson \& Johnson Prod LLC, Spring House, Pa 19477, USA

J Pharm Pharmacol 200456 (6) 749

Structural analysis of naphthoquinone protein adducts with liquid chromatography/tandem mass spectrometry and the scoring algorithm for spectral analysis (SALSA)

Zhang F, Bartels MJ// Dow Chem Co, Toxicol \& Environ Res \& Consulting, 1803 Bldg, Midland, Mi 48674, USA

Rapid Commun Mass Spectrom 200418 (16) 1809

Screening for topoisomerase I binding compounds by high-performance liquid chromatography-mass spectrometry

Zhang $\mathrm{H}, \mathrm{Gu} \mathrm{Q}$, Liang XL, Pan YJ*// *Zhejiang Univ, Dept Chem, Hangzhou, Peoples Rep China

Anal Biochem 2004329 (2) 173

High-throughput sample preparation procedures for the quantitation of a new bone integrin $\alpha_{v} \beta_{3}$ antagonist in human plasma and urine using liquid chromatography-tandem mass spectrometry

Zhang J, Zeng W, Kitchen C, Wang AQ, Musson DG// Merck Res Labs, WP75A-303, West Point, Pa 19486, USA

J Chromatogr B 2004806 (2) 167

Simultaneous determination of Z-SU5416 and its interconvertible geometric $E$-isomer in rat plasma by LC/MS/MS

Zhao YP, Sukbuntherng J, Antonian L*// *Sugen Inc, Dept Bioanal Metab \& Pharmacokinet, 230 East Grand Ave, San Francisco, Ca 94080, USA

J Pharm Biomed Anal 200435 (3) 513

Characterization of recombinant human granulocyte colony stimulating factor (rHuG-CSF) by capillary zone electrophoresis, capillary isoelectric focusing electrophoresis and electrospray ionization mass spectrometry

Zhou GH, Luo GA, Sun GQ, Cao YC, Zhang XD, Zhang X// Huadong Res Inst Med \& Biotech, 293 Zhongshan East Rd, CN-210002 Nanjing, Jiangsu, Peoples Rep China
J Pharm Biomed Anal 200435 (3) 425

Rapid ESI-MS method for examining the thermal decomposition of pharmaceuticals

Zhou W, Gilpin R*// *Wright State Univ, Coll Sci \& Math, Dayton, Oh 45435, USA

J Pharm Sci 200493 (6) 1545

\section{NATURAL PRODUCTS}

Detection of 2,4,6-trichloroanisole in microorganism-free irradiated raisins by solid-phase micro extraction and GC-MS

Aung LH, Jenner JF// USDA/ARS, Postharvest Qual \& Genet Res Unit, 9611 Sth Riverbend Ave, Parlier, Ca 93648, USA

J Stored Prod Res 200440 (4) 451

Confirmation of the identity of the carotenoids of tropical fruits by HPLCDAD and HPLC-MS

Azevedo-Meleiro CH, Rodriguez-Amaya DB*// *Univ Estadual Campinas, Fac Engn Alimentos, Dept Ciencia Alimentos, CP 6121, BR-13083-970 Campinas, SP, Brazil

J Food Compos Anal 200417 (3-4) 385

Mass spectrometric analysis of surfactant metabolism in human volunteers using deuteriated choline

Bernhard W, Pynn CJ, Jaworski A, Rau GA, Hohlfeld LM, Freihorst J, Poets CF, Stoll D, Postle AD// Univ Tubingen, Fac Med, Dept Neonatol, Calwer Str 7, DE-72076 Tubingen, Germany

Am J Respir Crit Care Med 2004170 (1) 54

Probing genetic variation and glycoform distribution in lectins of the Erythrina genus by mass spectrometry

Bonneil E, Young NM, Lis H, Sharon N, Thibault P*// *Natl Res Council Canada, Inst Biol Sci, 100 Sussex Dr, Ottawa, Ontario, Canada K1A 0R6

Arch Biochem Biophys 2004426 (2) 241

Identification and quantification of astaxanthin esters in shrimp (Pandalus borealis) and in a microalga (Haematococcus pluvialis) by liquid chromatography mass spectrometry using negative ion atmospheric pressure chemical ionization

Breithaupt DE// Univ Hohenheim, Inst Lebensmittelchem, Garbenstr 28, DE-70599 Stuttgart, Germany

J Agric Food Chem 200452 (12) 3870

Characterization of soluble non-covalent complexes between bovine serum albumin and $\beta$-1,2,3,4,6-penta- $O$-galloyl-D-glucopyranose by MALDI-TOF MS Chen YM, Hagerman AE*//*Miami Univ, Dept Chem \& Biochem, Oxford, Oh 45056, USA

J Agric Food Chem 200452 (12) 4008

Non-invasive online detection of nitric oxide from plants and some other organisms by mass spectrometry

Conrath U, Amoroso G, Kohle H, Sultemeyer DF// Univ Bonn, Inst Cellular \& Mol Bot, Plant Mol Biol Dept, 1 Kirschallee, DE-53115 Bonn, Germany Plant J 200438 (6) 1015

Structural characterization of fish egg vitelline envelope proteins by mass spectrometry

Darie CC, Biniossek ML, Jovine L, Litscher ES, Wassarman PM*// *UNY Mt Sinai Sch Med, Brookdale Dept Mol Cell \& Dev Biol, 1 Gustave L Levy Pl, New York, NY 10029, USA

Biochemistry 200443 (23) 7459

A simple, rapid and sensitive method for determination of aldehydes in human blood by gas chromatography/mass spectrometry and solid-phase microextraction with on-fiber derivatization

Deng C, Zhang X*// *Fudan Univ, Dept Chem, CN-200433 Shanghai, China Rapid Commun Mass Spectrom 200418 (15) 1715

Rapid determination of acetone in human plasma by gas chromatographymass spectrometry and solid-phase microextraction with on-fiber derivatization

Deng $\mathrm{CH}$, Zhang W, Zhang J, Zhang XM*// *Address as above J Chromatogr B 2004805 (2) 235

MALDI-TOFMS compared with other polyphasic taxonomy approaches for the identification and classification of Bacillus pumilus spores

Dickinson DN, La Duc MT, Satomi M, Winefordner JD, Powell DH, Venkateswaran K// Univ Florida, Dept Chem, POB 117200, Gainesville, Fl 32611, USA

J Microbiol Methods 200458 (1) 1

Analytical procedure for the in-vial derivatization-extraction of phenolic acids and flavonoids in methanolic and aqueous plant extracts followed by gas chromatography with mass-selective detection

Fiamegos YC, Nanos CG, Vervoort J, Stalikas CD*//*Univ Ioannina, Analyt Chem Lab, Dept Chem, GR-45110 Ioannina, Greece

J Chromatogr A 20041041 (1-2) 11 
Fast determination of the total free resveratrol content in wine by direct-exposure-probe, positive-ion chemical ionization and collision-induced-dissociation mass spectrometry

Flamini R, Della Vedova A// Inst Sperimentale Viticoltura, Lab Chim, Viale XXVIII Aprile 26, IT-26-31015 Conegliano, TV, Italy

Rapid Commun Mass Spectrom 200418 (17) 1925

Feeding fish with diets of different ratios of $\mathrm{C}_{3}$ - and $\mathrm{C}_{4}$-plant-derived ingredients: A laboratory analysis with implications for the back calculation of diet from stable isotope data

Focken U// Univ Hohenheim - 480B, Dept Aquacult Syst \& Anim Nutr Trop Subtrop, DE-70593 Stuttgart, Germany

Rapid Commun Mass Spectrom 200418 (18) 2087

Simultaneous determination of endogenous and ${ }^{13} \mathrm{C}$-labelled thyroid hormones in plasma by stable isotope dilution mass spectrometry

Hantson AL, De Meyer M, Guerit N// Fac Polytech Mons, Appl Chem \& Biochem Dept, 56 rue Epargne, BE-7000 Mons, Belgium

J Chromatogr B 2004807 (2) 185

Identification of monomenthyl succinate, monomenthyl glutarate, and dimenthyl glutarate in nature by high performance liquid chromatography-tandem mass spectrometry

Hiserodt RD, Adedeji J, John TV, Dewis ML// Int Flavors \& Fragrances Inc, Res \& Dev, 1515 State Highway 36, Union Beach, NJ 07735, USA

J Agric Food Chem 200452 (11) 3536

Rapid and sensitive screening for and chemical diagnosis of Canavan disease by gas chromatography-mass spectrometry

Inoue Y, Kuhara $\mathrm{T} * / / *$ Kanazawa Med Univ, Med Res Inst, Div Human Genet, 1-1 Daigaku, Uchinada, Ishikawa 920 029, Japan

J Chromatogr B 2004806 (1) 33

Determination of the ${ }^{13} \mathrm{C} /{ }^{12} \mathrm{C}$ ratio of ethanol derived from fruit juices and maple syrup by isotope ratio mass spectrometry: Collaborative study

Jamin E, Martin F, Martin GG// Eurofins Sci Analyt, BP42301, rue Pierre Adolphe Bobierre, FR-44323 Nantes 3, France

J AOAC Int 200487 (3) 621

Analysis of microsomal metabolic stability using high-flow-rate extraction coupled to capillary liquid chromatography-mass spectrometry

Laven A, Markides $\mathrm{K}^{*}$, Langstrom B// *Uppsala Univ, Inst Chem, Dept Analyt Chem, Box 599, SE-75124 Uppsala, Sweden

J Chromatogr B 2004806 (2) 119

Solid phase extraction procedure for urinary organic acid analysis by gas chromatography mass spectrometry

Liu AP, Kushnir MM, Roberts WL, Pasquali M// ARUP Inst Clin \& Expt Pathol, 500 Chipeta Way, Salt Lake City, Ut 84108, USA

J Chromatogr B 2004806 (2) 283

Host-defence skin peptides of the Australian Common Froglet Crinia signifera: Sequence determination using positive and negative ion electrospray mass spectra

Maselli VM, Brinkworth CS, Bowie $\mathrm{JH}^{*}$, Tyler MJ// *Univ Adelaide, Dept Chem, Adelaide, SA 5005, Australia

Rapid Commun Mass Spectrom 200418 (18) 2155

Mass spectrometric imaging of highly curved membranes during Tetrahymena mating

Ostrowski SG, Van Bell CT, Winograd N, Ewing AG// Penn State Univ, Dept Chem, University Park, Pa 16802, USA

Science 2004305 (5680) 71

Identification and quantification of polyphenols in carob fruits (Ceratonia siliqua L.) and derived products by HPLC-UV-ESI/MS

Papagiannopoulos M, Wollseifen HR, Mellenthin A, Haber B, Galensa R// Univ Bonn, Dept Food Sci \& Food Chem, Endenicher Allee 11-13, DE-53115 Bonn, Germany

J Agric Food Chem 200452 (12) 3784

Separation and characterization of phenolic compounds in fennel (Foeniculum vulgare) using liquid chromatography-negative electrospray ionization tandem mass spectrometry

Parejo I, Jauregui O, Sanchez-Rabaneda F, Viladomat F, Bastida J, Codina C*// *Univ Barcelona, Fac Farm, Dept Nat Prod, Avda Joan XXIII s/n, ES-08028 Barcelona, Spain

J Agric Food Chem 200452 (12) 3679

Determination of alkanolamines in cattails (Typha latifolia) utilizing electrospray ionization with selected reaction monitoring and ion-exchange chromatography

Peru KM, Headley JV*, Doucette WJ// *Environm Canada, Nat Water Res Inst, 11 Innovation Blvd, Saskatoon, Saskatchewen, Canada S7N 3H5 Rapid Commun Mass Spectrom 200418 (14) 1629

The Schiff base at gossypol with $3,6,9,12,15,18,21,24$-octaoxa-pentacosylamime complexes and monovalent cations studied by electrospray ionization-mass spectrometry, ${ }^{1} \mathrm{H}$ nuclear magnetic resonance, Fourier transform infrared, as well as PM5 semiempirical methods
Przybylski P, Brzezinski B*, Bartl F// *Adam Mickiewicz Univ Poznan, Fac Chem, Grunwaldzka 6, PL-60780 Poznan, Poland

Biopolymers 200474 (4) 273

Structural investigations of flavonol glycosides from sea buckthorn (Hippophae rhamnoides) pomace by NMR spectroscopy and HPLC-ESI$\mathrm{MS}^{\mathrm{n}}$

Rosch D, Krumbein A, Mugge C, Kroh LW*// *Tech Univ Berlin, Inst Lebensmitteltechnol \& Lebensmittelchem, Gustav Meyer Allee 25, DE13355 Berlin, Germany

J Agric Food Chem 200452 (13) 4039

Determination of endogenous and supplied deuterated abscisic acid in plant tissues by high-performance liquid chromatography-electrospray ionization tandem mass spectrometry with multiple reaction monitoring

Ross ARS, Ambrose SJ, Cutler AJ, Feurtado JA, Kermode AR, Nelson K, Zhou R, Abrams SR*// *Natl Res Council Canada, Inst Plant Biotechnol, 110 Gymnasium Rd, Saskatoon, Saskatchewan, Canada S7N 0W9 Anal Biochem 2004329 (2) 324

Rapid identification of environmental bacterial strains by matrix-assisted laser desorption/ionization time-of-flight mass spectrometry

Ruelle V, El Moualij B, Zorzi W, Ledent P, De Pauw E// Univ Liege, Mass Spectrom Lab, 3 Allee de la Chimie, B6c, BE-4000 Liege, Belgium Rapid Commun Mass Spectrom 200418 (18) 2013

New dibenzotropolone derivatives characterized from black tea using LC/ MS/MS

Sang SM, Tian SY, Stark RE, Yang CS, Ho CT// Rutgers State Univ, Ernest Mario Sch Pharm, Dept Biol Chem, 164 Frelinghuysen Rd, Piscataway, NJ 08854, USA

Bioorg Med Chem 200412 (11) 3009

Identification and quantification of caffeoylquinic acids and flavonoids from artichoke (Cynara scolymus L.) heads, juice, and pomace by HPLC-DAD$\mathrm{ESI} / \mathrm{MS}^{\mathrm{n}}$

Schutz K, Kammerer D, Carle R, Schieber A*// *Univ Hohenheim, Inst Food Technol, Sect Plant Foodstuff Technol, August von Hartmann Str 3, DE-70599 Stuttgart, Germany

J Agric Food Chem 200452 (13) 4090

Capillary electrophoresis and mass spectrometry for screening of metabolic disorders in newborns

Senk P, Kozak L, Foret F*// *Inst Analyt Chem, Veveri 97, CZ-61142 Brno, Czech Republic

Electrophoresis 200425 (10-11) 1447

Mass spectrometric analysis of posttranslational modifications of a carrot extracellular glycoprotein

Shang CW, Shibahara T, Hanada K, Iwafune Y, Hirano H*// *Yokohama City Univ, Grad Sch Integrated Sci, Kihara Inst Biol Res, Maioka cho 641-12, Yokohama, Kanagawa 244 081, Japan

Biochemistry 200443 (20) 6281

Isotope dilution tandem mass spectrometric method for $\mathrm{T}_{4} / \mathrm{T}_{3}$

Soukhova N, Soldin OP, Soldin SJ*//*Georgetown Clin Res Ctr, Bioanalyt Core Lab, GM-12A Preclin Sci Bldg, 3900 Reservoir Rd NW, Washington, DC 20007, USA

Clin Chim Acta 2004343 (1-2) 185

Application of electrospray ionization mass spectrometry in rapid typing of fengycin homologues produced by Bacillus subtilis

Wang J, Liu J, Wang X, Yao J, Yu Z// Chinese Acad Sci, Key Lab Ion Beam Bioengn, CN-230031 Hefei, Anhui, Peoples Rep China

Lett Appl Microbiol 200439 (1) 98

\section{ANALYSIS OF ORGANIC COMPOUNDS}

Stability of the porphyrin-calix[4]arene complexes analysed by electrospray ionization mass spectometry

Arai S, Ishihara S, Takeoka S, Ohkawa H, Shibue T, Nishide $\mathrm{H} * / / *$ Waseda Univ, Sch Sci Engn, Dept Appl Chem, 3-4-1 Okubo, Shinjuku, Tokyo 169 8555, Japan

Rapid Commun Mass Spectrom 200418 (18) 2065

Generation and characterization of ionic and neutral methylene isothiocyanate by a combined tandem mass spectrometry and computational study

Nagi Reddy P, Srikanth R, Bhanuprakash $\mathrm{K}^{*}$, Srinivas R// *Indian Inst Chem Technol, Inorgan Chem Div, IN-500007 Hyderabad, India Rapid Commun Mass Spectrom 200418 (17) 1939

Mass spectral study of meso-alkyl and meso-cycloalkyl calix(4)pyrroles under electron impact conditions

Prabhakar S, Radha Kishan M, Mirza SP, Raghavan KV, Vairamani M// Indian Inst Chem Tech, Nat Ctr Mass Spectrom, IN-500007 Hyderabad, India Rapid Commun Mass Spectrom 200418 (18) 2077

Trimethylsilyl transfer during electron ionization mass spectral fragmenta- 
tion of some $\omega$-hydroxycarboxylic and $\omega$-dicarboxylic acid trimethylsilyl derivatives and the effect of chain length

Rontani JF, Aubert C// Ctr Oceanol Marseille - OSU, Lab Microbiol Geochim \& Ecol Marines, UMR 6117, Campus Luminy - Case 901, FR-13288 Marseille, France

Rapid Commun Mass Spectrom 200418 (17) 1889

Probing the molecular weight distributions of non-boiling petroleum fractions by $\mathrm{Ag}^{+}$electrospray ionization mass spectrometry

Roussis SG, Proulx R// Imperial Oil, Prod \& Chem Div, Res Dept, 453 Christina St Sth, PO Box 3022, Sarnia, Ontario, Canada N7T 8C8

Rapid Commun Mass Spectrom 200418 (15) 1761

Fragmentation study of diastereoisomeric 2-methyltetrols, oxidation products of isoprene, as their trimethylsilyl ethers, using gas chromatography/ion trap mass spectrometry

Wang W, Vas G, Dommisse R, Loones K, Claeys M*// *Univ Antwerp, Dept Pharmaceut Sci, Campus Drie Eiken, Universiteitspl 1, BE-2610 Antwerp, Belgium

Rapid Commun Mass Spectrom 200418 (16) 1787

Identification of gasoline soot in suspect arson cases by using headspace solid phase microextraction-GC/MS

Wu CH, Chen CL, Huang CT, Lee MR*, Huang CM// *Natl Chung Hsing Univ, Dept Chem, Taichung, Taiwan

Anal Lett 200437 (7) 1373

Electron-ionization-induced mass spectral study of isomeric (E)- $N$-ethyl-1,2bispyridylethylene iodides (Letter)

Wybieralska J// Adam Mickiewicz Univ, Fac Chem, Dept Mass Spectrom Organ Cpds, Grunwaldzka 6, PL-60780 Poznan, Poland

Rapid Commun Mass Spectrom 200418 (17) 2008

Effect of matrix and solvent on the analysis of novel poly(phenylenevinylene) derivatives by matrix-assisted laser desorption/ionization time-offlight mass spectometry

Zhang Z, Deng H*, Deng Q, Zhao S// *Sun Yat-Sen Univ, Instrum Anal \& Res Ctr, CN-510275 Guangzhou, Guangdong, China

Rapid Commun Mass Spectrom 200418 (18) 2146

\section{ANALYSIS OF INORGANICS/ORGANOMETALLICS}

Electrospray ionization mass spectrometry in the structural characterization of some diphenyllead(VI) thiosemicarbazonates

Casas JS, Garcia-Tasende MS, Sordo J, Taboada C, Tubaro M, Traldi P*, Vidarte MJ// *CNR, Inst Sci \& Technol Mol, Corso Stati Uniti 4, IT-35100 Padua, Italy

Rapid Commun Mass Spectrom 200418 (16) 1856

\section{SURFACE ANALYSIS}

Depth profiling of 4-acetamindophenol-doped poly(lactic acid) films using cluster secondary ion mass spectrometry

Mahoney CM, Roberson SV, Gillen G// NIST, Chem Sci \& Technol Lab, Surface \& Microanal Sci Div, 100 Bureau Dr, Mail Stop 8371, Gaithersburg, Md 20899, USA

Anal Chem 200476 (11) 3199

Characterization of the surface of conventional hydrogel and silicone hydrogel contact lenses by time-of-flight secondary ion mass spectrometry Maldonado-Codina C, Morgan PB, Efron N, Canry JC// UMIST, Dept Optom \& Neurosci, Eurolens Res, POB 88, Manchester, M60 1QD, England Optom Vis Sci 200481 (6) 455

\section{ENVIRONMENTAL ANALYSIS}

Determination of thiabendazole in orange juice and rind by liquid chromatography with fluorescence detection and confirmation by gas chromatography/mass spectrometry after extraction by matrix solid-phase dispersion Albero B, Sanchez-Brunete C, Tadeo JL*// *INIA, Dept Med Ambiente, Apdo 8111, ES-28080 Madrid, Spain

J AOAC Int 200487 (3) 664

Electron ionization mass spectrometric study of antifungal 1,2,4-trithiols (Letter)

Alvarez C, Penieres G, Cruz T, Londono A, Velasco B, Miranda R*// *Fac Estudios Superiores Cuautitlan-UNAM, Avda Primero Mayo s/n, MX-54740 Cuautitlan Izcalli, Edo de Mexico, Mexico

Rapid Commun Mass Spectrom 200418 (14) 1660

Influence of flooding on $\delta^{15} \mathrm{~N}, \delta^{18} \mathrm{O},{ }^{1} \delta^{15} \mathrm{~N}$ and ${ }^{2} \delta^{15} \mathrm{~N}$ signatures of $\mathrm{N}_{2} \mathrm{O}$ released from estuarine soils - A laboratory experiment using tidal flooding chambers

Bol R, Rockmann T, Blackwell M, Yamulki S// Nth Wyke Res Station, Inst
Grassland \& Environm Res, Okehampton EX20 2SB, England Rapid Commun Mass Spectrom 200418 (14) 1561

Determination of abamectin in soil samples using high-performance liquid chromatography with tandem mass spectrometry

Brewer BN, Armbrust KL, Mead KT, Holmes WE*// *Mississippi State Chem Lab, Box CR, Mississippi State, Ms 39762, USA

Rapid Commun Mass Spectrom 200418 (15) 1693

Comparison chromatography of accelerator mass spectrometry with gas for the determination of pesticide residues in individual items in the diets of wild birds and mammals

Brown P, Garner C, Glass R, Ridgway C, Hart A// Cent Sci Lab, York YO41 1LZ, England

J Agric Food Chem 200452 (12) 3693

Determination of pharmaceutical compounds in surface- and ground-water samples by solid-phase extraction and high-performance liquid chromatography-electrospray ionization mass spectrometry

Cahill JD, Furlong ET*, Burkhardt MR, Kolpin D, Anderson LG// *US Geol Survey, Natl Water Qual Lab, Denver Fed Ctr, POB 25046, MS 407, Denver, Co 80225, USA

J Chromatogr A 20041041 (1-2) 171

Determination of acrylamide in drinking water by large-volume direct injection and ion-exclusion chromatography-mass spectrometry

Cavalli S, Polesello S*, Saccani G// *IRSA, CNR, Via Mornera 25, IT-20047 Brugherio, MI, Italy

J Chromatogr A 20041039 (1-2) 155

Validation of a real-time monitoring method for aniline in freshwater by highperformance liquid chromatography on porous graphitic carbon/electrospray ionization tandem mass spectrometry

Delepee R, Chaimbault P, Antignac JP, Lafosse M// Univ Orleans, Inst Chim Organ \& Anal, UMR 6005, rue Chartres, BP 6759, FR-45067 Orleans, France Rapid Commun Mass Spectrom 200418 (14) 1548

Improved separation of the 209 polychlorinated biphenyl congeners using comprehensive two-dimensional gas chromatography-time-of-flight mass spectrometry

Focant JF, Sjodin A, Patterson DG// Univ Liege, Mass Spectrometry Lab, Allee Chim 3, B-6c Sart Tilman, BE-4000 Cointe Ougree, Belgium J Chromatogr A 20041040 (2) 227

The effect of $\mathrm{N}_{2} \mathrm{O}$ on the isotopic composition of air- $\mathrm{CO}_{2}$ samples

Ghosh P, Brand WA*// *Max-Planck-Inst Biogeochem, PO Box 100164, DE-07701 Jena, Germany

Rapid Commun Mass Spectrom 200418 (16) 1830

Measurement of perchlorate in water by use of an ${ }^{18} \mathrm{O}$-enriched isotopic standard and ion chromatography with mass spectrometric detection

Hedrick E, Munch D// US EPA, 26 West Martin Luther King Dr, Cincinnati, Oh 45268, USA

J Chromatogr A 20041039 (1-2) 83

Rapid determination of chlorinated pesticides in fish by freezing-lipid filtration, solid-phase extraction and gas chromatography-mass spectrometry

Hong J, Kim HY, Kim DG, Seo J, Kim KJ// Korea Basic Sci Inst, Hazardous Subst Res Team, Anam 5 Ga, Seoul 136 701, South Korea

J Chromatogr A 20041038 (1-2) 27

Determination of pesticides in soil by liquid-phase microextraction and gas chromatography-mass spectrometry

Hou L, Lee HK*// *Natl Univ Singapore, Dept Chem, 3 Sci Dr 3, SG-117543

Singapore, Rep Singapore

J Chromatogr A 20041038 (1-2) 37

On-line laser desorption/ionization mass spectrometry of matrix-coated aerosols

Jackson SN, Mishra S, Murray KK*// *Louisiana State Univ, Dept Chem, Baton Rouge, La 70803, USA

Rapid Commun Mass Spectrom 200418 (18) 2041

Simultaneous extraction of tetracycline, macrolide and sulfonamide antibiotics from agricultural soils using pressurised liquid extraction, followed by solidphase extraction and liquid chromatography-tandem mass spectrometry

Jacobsen AM, Halling-Sorensen B, Ingerslev F, Hansen SH// Danish Univ Pharmaceut Sci, Dept Analyt Chem, Universitetsparken, DK-2100 Copenhagen, Denmark

J Chromatogr A 20041038 (1-2) 157

Trace analysis of phenolic xenoestrogens in water samples by stir bar sorptive extraction with in situ derivatization and thermal desorption-gas chromatography-mass spectrometry

Kawaguchi M, Inoue K, Yoshimura M, Sakui N, Okanouchi N, Ito R, Yoshimura Y, Nakazawa H*// *Hoshi Univ, Fac Pharmaceut Sci, Dept Analyt Chem, 2-4-41 Ebara, Shinagawa ku, Tokyo 142 8501, Japan

J Chromatogr A 20041041 (1-2) 19

Kel-FTM discs improve storage time of canopy air samples in $10-\mathrm{mL}$ vials for 
$\mathrm{CO}_{2}-\delta^{13} \mathrm{C}$ analysis (Letter)

Knohl A, Werner RA, Geilmann H, Brand WA// Univ Calif, Dept Environ Sci Policy Management, 151 Hilgard Hall 3110, Berkeley, Ca 94720, USA Rapid Commun Mass Spectrom 200418 (14) 1663

Evaluation of ${ }^{14} \mathrm{C}$ abundance in soil respiration using accelerator mass spectrometry

Koarashi J, Iida T, Moriizumi J, Asano T// Japan Nucl Cycle Dev Inst, Radiat Prot Div, Ibaraki 319 1194, Japan

J Environ Radioact 200475 (2) 117

Microwave-assisted extraction followed by gas chromatography-mass spectrometry for the determination of endocrine disrupting chemicals in river sediments

Liu R, Zhou JL*, Wilding A// *Univ Sussex, Sch Life Sci, Dept Biol \& Environm Sci, Brighton BN1 9QJ, England

J Chromatogr A 20041038 (1-2) 19

Determination of trace-level haloacetic acids in drinking water by ion chromatography-inductively coupled plasma mass spectrometry

Liu YJ, Mou SF*, Chen DY// *Chinese Acad Sci, Ecoenvironm Sci Res Ctr, POB 2871, CN-100085 Beijing, Peoples Rep China

J Chromatogr A 20041039 (1-2) 89

Determination of amines as pentafluoropropionic acid anhydride derivatives in biological samples using liquid chromatography and tandem mass spectrometry

Marand A, Karlsson D, Dalene M, Skarping G*//*Stockholm Univ, Work Environm Chem, POB 460, SE-28124 Hassleholm, Sweden

Analyst 2004129 (6) 522

Simultaneous determination of alkylphenols and bisphenol A in river water by stir bar sorptive extraction with in situ acetylation and thermal desorption-gas chromatography-mass spectrometry

Nakamura S, Daishima S// Yokogawa Analyt Syst Inc, 9-1 Takakura cho, Hachioji, Tokyo 192 0033, Japan

J Chromatogr A 20041038 (1-2) 291

Determination of toxic cyclic heptapeptides by liquid chromatography with detection using ultra-violet, protein phosphatase assay and tandem mass spectrometry

Ortea PM, Allis O, Healy BM, Lehane M, Ni Shuilleabhain A, Furey A, James KJ*// *Cork Inst Technol, Dept Chem, Mass Spect Ctr Proteom \& Biotoxin Res, Cork, Rep Ireland

Chemosphere 200455 (10) 1395

Potential of liquid chromatography-atmospheric pressure chemical ionisation tandem mass spectrometry for determination of fosetyl-aluminium residues in dried hops

Poustka J, Hajslova J, Holadova K, Novakova K// Inst Chem Technol, Dept Food Chem \& Anal, Technicka 3, CZ-16628 Prague 6, Czech Republic

Czech J Food Sci 200422 (1) 24

Determination of organophosphorus pesticides using membrane-assisted solvent extraction combined with large volume injection-gas chromatographymass spectrometric detection

Schellin M, Hauser B, Popp P// UFZ Ctr Environm Res, Dept Analyt Chem, Permoserstr 15, DE-04318 Leipzig, Germany

J Chromatogr A 20041040 (2) 251

Is the isotopic composition of nitrous oxide an indicator for its origin from nitrification or dentrification? A theoretical approach from referred data and microbiological and enzyme kinetic aspects

Schmidt HL, Werner RA, Yoshida N, Well R// Prielhofweg 2, DE-84036 Landshut, Germany

Rapid Commun Mass Spectrom 200418 (18) 2036

$\mathrm{N}_{2} \mathrm{O}$ influence on isotopic measurements of atmospheric $\mathrm{CO}_{2}$

Sirignano C, Neubert REM, Meijer HAJ// Rijksuniversiteit Groningen, Ctr Isotope Res - CIO, Nijenborgh 4, NL-9747 AG Groningen, The Netherlands

Rapid Commun Mass Spectrom 200418 (16) 1839

Quantification of hydrogen cyanide in humid air by selected ion flow tube mass spectrometry

Spanel P, Wang T, Smith D*// *Keele Univ, Sch Med, Inst Sci \& Technol Med, Thornburrow Dr, Hartshill, Stoke-on-Trent ST4 7QB, England

Rapid Commun Mass Spectrom 200418 (16) 1869

Determination of residues of propamocarb in wine by liquid chromatographyelectrospray mass spectrometry with direct injection

Taylor JC, Hird SJ, Sykes MD, Startin JR*// *Cent Sci Lab, York YO41 1LZ, England

Food Addit Contam 200421 (6) 572

A portable automated system for trace gas sampling in the field and stable isotope analysis in the laboratory

Theis DE, Saurer M, Blum H, Frossard E, Seigwolf RTW*// *Paul Scherrer Inst, Lab Atmos Chem, CH-5232 Villigen, Switzerland

Rapid Commun Mass Spectrom 200418 (18) 2106
Determination of molecular formulas and structural regularities of low molecular weight fulvic acids by size-exclusion chromatography with electrospray ionization quadrupole time-of-flight mass spectrometry

These A, Winkler M, Thomas C, Reemtsma T*//*Tech Univ Berlin, Dept Water Qual Control, Sekr KF4, Str 17 Juni 135, DE-10623 Berlin, Germany Rapid Commun Mass Spectrom 200418 (16) 1777

Ultrafine nitrate particle events in Baltimore observed by real-time single particle mass spectrometry

Tolocka MP, Lake DA, Johnston MV*, Wexler AS// *Univ Delaware, Dept Chem \& Biochem, Brown Lab 107, Newark, De 19716, USA

Atmos Environ 200438 (20) 3215

Liquid chromatography-mass spectrometry analysis of hydroxylated polycyclic aromatic hydrocarbons, formed in a simulator of the human gastrointestinal tract

Van de Wiele TR, Peru KM, Verstraete W, Siciliano SD, Headley JV*// *Natl Water Res Inst, 11 Innovation Blvd, Saskatoon, SK, Canada S7N 3H5 J Chromatogr B 2004806 (2) 245

Determination of organophosphorus pesticides in water by HPLC-MS-MS (German, English Abstract)

Ventker $\mathrm{M}$, Werres $\mathrm{F}^{*}$, Balsaa $\mathrm{P}$, Winterhalter $\mathrm{P}$, Overath $\mathrm{H} / /$ *Inst Gerhard-Mercator GH Univ Duisburg, IWW Rheinisch Westfalisches, Inst Wasserforsch Gemeinnutzige GmbH, Moritzstr 26, DE-45476 Mulheim an der Ruhr, Germany

Acta Hydrochim Hydrobiol 200432 (1) 40

Solid-phase extraction-high-performance liquid chromatography-ion trap mass spectrometry for analysis of trace concentrations of macrolide antibiotics in natural and waste water matrices

Yang S, Carlson $\mathrm{KH}^{*} / / *$ Colorado State Univ, Dept Civil \& Environm Engn, Fort Collins, Co 80523, USA

J Chromatogr A 20041038 (1-2) 141

Quantitative determination of trace concentrations of tetracycline and sulfonomide antibiotics in surface water using solid phase extraction and liquid chromatography/ion trap tandem mass spectrometry

Yang S, Cha J, Carlson $\mathrm{K} * / / *$ Address as above

Rapid Commun Mass Spectrom 200418 (18) 2131

\section{ELEMENTAL ANALYSIS}

Multivariate pattern matching of trace elements in solids by laser ablation inductively coupled plasma-mass spectrometry: Source attribution and preliminary diagnosis of fractionation

Aeschliman DB, Bajic SJ, Baldwin DP, Houk RS*// *Iowa State Univ, Dept Chem, US Dept Energy, Ames Lab, Ames, Ia 50011, USA

Anal Chem 200476 (11) 3119

Determination of anthropogenic input of $\mathrm{Ru}, \mathrm{Rh} \mathrm{Pd}, \mathrm{Re}, \mathrm{Os}, \mathrm{Ir}$ and $\mathrm{Pt}$ in soils along Austrian motorways by isotope dilution ICP-MS

Fritsche J, Meisel T*// *Univ Leoben, Franz Josef Str 18, AT-8700 Leoben, Austria

Sci Total Environ 2004325 (1-3) 145

Determination of intratest variability of trace elements in foraminifera by laser ablation inductively coupled plasma-mass spectrometry

Hathorne EC, Alard O, James RH, Rogers NW// Open Univ, Dept Earth Sci, Walton Hall, Milton Keynes MK7 6AA, England

Geochem Geophys Geosyst 200348408

Chemical resolution of $\mathrm{Pu}^{+}$from $\mathrm{U}^{+}$and $\mathrm{Am}^{+}$using a band-pass reaction cell inductively coupled plasma mass spectrometer

Tanner SD, Li SD, Vais V, Baranov VI, Bandura DR// PerkinElmer SCIEX, 71 Four Valley Dr, Concord, Ontario, Canada L4K 4V8

Anal Chem 200476 (11) 3042

Determination of elements in native and bypass human coronary artery plaque deposits from the same heart using inductively coupled plasma-mass spectrometry

Thiam S, Tracy RE, Robinson JW*, Warner IM// *Louisiana State Univ, Dept Chem, Baton Rouge, La 70803, USA

J Environ Sci Health A 200439 (6) 1497

Direct analysis of $\mathrm{Ca}, \mathrm{P}$, and $\mathrm{Fe}$ in oleochemicals by inductively coupled plasma MS

Wiedemann SCC, Abbes H, Hansen WG// Uniqema, Corp Analyt Dev, POB 2, NL-2800 AA Gouda, The Netherlands

J Am Oil Chem Soc 200481 (5) 437

Speciation of heavy metals in marine sediments from the East China Sea by ICP-MS with sequential extraction

Yuan CG, Shi JB, He B, Liu JF, Liang LN, Jiang GB*// *Chinese Acad Sci, Ecoenvironm Sci Res Ctr, Key Lab Environm Chem \& Ecotoxicol, POB 2871, CN-100085 Beijing, Peoples Rep China

Environ Int 200430 (6) 769 